\title{
Association between HIF-1a C1772T/G1790A polymorphisms and cancer susceptibility: an updated systematic review and meta-analysis based on 40 case-control studies
}

Qing Yan $^{\dagger}$, Pin Chen ${ }^{\dagger}$, Songtao Wang ${ }^{\dagger}$, Ning Liu, Peng Zhao and Aihua Gu*

\begin{abstract}
Background: HIF-1 (hypoxia-inducible factor 1) is a transcriptional activator that functions as a critical regulator of oxygen homeostasis. Recently, a large number of epidemiological studies have investigated the relationship between HIF-1a C1772T/G1790A polymorphisms and cancer susceptibility. However, the results remain inconclusive. Therefore, we performed a meta-analysis on all of the available case-control studies to systematically summarize the possible association.

Methods: A literature search was performed using PubMed and the Web of Science database to obtain relevant published studies. Pooled odds ratios (ORs) and corresponding 95\% confidence intervals (Cls) for the relationship between HIF-1a C1772T/G1790A polymorphisms and cancer susceptibility were calculated using fixed-and random-effects models when appropriate. Heterogeneity tests, sensitivity analyses and publication bias assessments were also performed in our meta-analysis.

Results: A total of 40 studies met the inclusion criteria were included in the meta-analysis: 40 studies comprised of 10869 cases and 14289 controls for the HIF-1a C1772T polymorphism and 30 studies comprised of 7117 cases and 10442 controls for the HIF-1a G1790A polymorphism. The results demonstrated that there were significant association between the HIF-1a C1772T polymorphism and cancer susceptibility under four genetic models ( $T$ vs. $C C: \mathrm{OR}=1.63,95 \% \mathrm{Cl}=1.02-2.60 ; \mathrm{CT}+\Pi$ vs. $\mathrm{CC}: \mathrm{OR}=1.15,95 \% \mathrm{Cl}=1.01-1.34 ; \pi$ vs. $\mathrm{CT}+\mathrm{CC}: \mathrm{OR}=2.11$, $95 \% \mathrm{Cl}=1.32-3.77$; $T$ vs. C: $\mathrm{OR}=1.21,95 \% \mathrm{Cl}=1.04-1.41)$. Similarly, the statistically significant association between the HIF-1a G1790A polymorphism and cancer susceptibility was found to be consistently strong in all of the genetic models. Moreover, increased cancer risk was observed when the data were stratified by cancer type, ethnicity and the source of controls.

Conclusions: This meta-analysis demonstrates that both the C1772T and G1790A polymorphisms in the HIF-1a gene likely contribute to increased cancer susceptibility, especially in the Asian population and in breast cancer, lung cancer, pancreatic cancer and oral cancer. However, further research is necessary to evaluate the relationship between these polymorphisms and cancer risk.
\end{abstract}

Keywords: HIF-1 gene, Polymorphism, Cancer, Susceptibility, Meta-analysis

\footnotetext{
*Correspondence: zhaopeng@njmu.edu.cn; aihuagu@njmu.edu.cn

${ }^{\dagger}$ Equal contributors

Department of Neurosurgery, The First Affiliated Hospital, Nanjing Medical

University, 300 Guangzhou Road, Nanjing 210029, China
} 


\section{Background}

Human cancer is a major cause of death in the world, and it is estimated that the number of new cases will increase to more than 15 million in the coming decade, creating a substantial worldwide public health burden $[1,2]$. Various factors, such as genetic and environmental influences, are associated with cancer prognosis. However, the exact etiology and mechanism of carcinogenesis have not yet been clearly elucidated. In recent years, it has become well-accepted that intrinsic factors, such as host genetic susceptibility, may play important roles in the process of cancer development $[3,4]$, and an increasing number of studies have focused on the association between genetic factors and cancer susceptibility.

Hypoxia-inducible factor 1 (HIF-1) is a transcriptional activator that functions as a critical regulator of oxygen homeostasis. It is a heterodimer composed of two subunits, HIF- $1 \alpha$ and HIF- $1 \beta$, which dimerize and bind to DNA via the basic helix-loop-helix Per/Arnt/Sim (bHLH-PAS) domain $[5,6]$. HIF-1 $\alpha$ expression is induced in hypoxic cells, and its level exponentially increase when the cells are exposed to $\mathrm{O}_{2}$ concentration of less than $6 \%$. Under hypoxic condition, HIF- $1 \alpha$ ubiquitination decreases dramatically, resulting in an accumulation of the protein, while under normoxic condition, HIF- $1 \alpha$ is rapidly degraded through von Hippel-Lindau (VHL)-mediated ubiquitination and proteasomal degradation [7-10]. HIF-1 has also been suggested to play an important role in tumor development, progression and metastasis, and HIF-1 can activate the transcription of more than 60 target genes that are involved in crucial aspects of cancer establishment, including cell survival, glucose metabolism, angiogenesis and invasion $[11,12]$.

The HIF-1 $\alpha$ gene is located on chromosome 14q21-24, and recent studies have shown that there are a total of 35 common single nucleotide polymorphisms (SNPs) throughout the HIF-1 $\alpha$ gene in Caucasian and Asian population [13-15]. Two important SNPs in exon 12 of the HIF-1 gene, HIF-1 $\alpha$ C1772T (rs11549465) and HIF-1 $\alpha$ G1790A (rs11549467), lead to amino acid substitution of proline to serine at position 582 and alanine to threonine at position 588 of the protein, respectively $[8,16,17]$. These two polymorphisms have been demonstrated to be functionally meaningful, resulting in increased transcriptional activity of HIF-1 $\alpha[14,18]$. Previous studies have shown that the overexpression of HIF- $1 \alpha$ is significantly associated with cell proliferation, increased tumor susceptibility, tumor size, lymph node metastasis and prognosis $[19,20]$.

In recent years, the HIF-1 $\alpha$ gene has been a research focus in the scientific community, and many epidemiological studies have been performed to assess the association between HIF-1 $\alpha$ C1772T/G1790A polymorphisms and cancer susceptibility. However, the results of the different studies are conflicting. Hence, we performed a meta-analysis of all of the eligible studies to clarify the role of HIF-1 $\alpha$ C1772T/G1790A polymorphisms in cancer development.

\section{Methods}

\section{Study eligibility and validity assessment}

We performed a computerized literature search of the PubMed and Web of Science databases to identify all of the relevant studies of cancer that contained sufficient genotyping data for at least one of the two polymorphisms, HIF-1 $\alpha$ C1772T or HIF-1 $\alpha$ G1790A. The search strategy was designed by two researchers and included the following keywords: "HIF-1 OR hypoxia-inducible factor-1" and "polymorphism", and the last search was updated on September 20th, 2013. To obtain all eligible publications, we also manually reviewed the references of the selected articles to identify other potential eligible publications. Articles investigating the association between cancer risk and the HIF-1 $\alpha$ polymorphisms were identified with no language restriction.

\section{Inclusion criteria}

The studies selected were required to meet the following criteria: 1) evaluate the association between the HIF-1 $\alpha$ C1772T and/or HIF-1 $\alpha$ G1790A polymorphisms and cancer risk; 2) use a human case-control design; 3) contain sufficient published data for the estimation of an odds ratio (OR) with a $95 \%$ confidence interval (CI).

\section{Data extraction}

Data were extracted from all of the eligible publications by two investigators (Yan and Chen) independently, according to the inclusion criteria listed above. Disagreements between the two investigators were resolved by discussion until a consensus was reached. The following information was extracted from each of the included publications: the first author's name, publication data, country of origin, ethnicities of the sample population (categorised as Asians, Caucasians and Mixed), cancer type, source of control group (population- or hospital-based controls), total number of cases and controls, and the number of cases and controls with the HIF-1 $\alpha$ C1772T/G1790A polymorphisms.

\section{Statistical methods}

The strength of the association between the HIF- $1 \alpha$ C1772T/HIF-1 $\alpha$ G1790A polymorphisms and cancer risk was measured by ORs with $95 \%$ CIs. The statistical significance of the pooled OR was calculated by the $\mathrm{Z}$ test, a $P<0.05$ was considered to be statistically significant ( $P$-values were two sided). For HIF-1 $\alpha$ C1772T polymorphism, we examined the overall ORs and compared the cancer incidence using the allelic model ( $T$ versus $C$ ), homozygote model (TT versus $\mathrm{CC}$ ), heterozygote model 
(TC versus $\mathrm{CC}$ ), dominant model ( $\mathrm{TT}+\mathrm{TC}$ versus $\mathrm{CC}$ ), recessive model (TT versus TC $+\mathrm{CC}$ ). For HIF-1 $\alpha$ G1790A polymorphism, we evaluated the risk in the allelic model (A versus $\mathrm{G}$ ), homozygote model (AA versus GG), heterozygote comparison model (GA versus GG), dominant models (AA + AG versus $G G$ ), and recessive model (AA versus $A G+G G)$. Subgroup analyses were also conducted by ethnicity, cancer type ("other cancer groups" means any cancer types with less than two separate publications) and source of controls. Statistical heterogeneity was estimated by a chi-square based Q-test, and when $P<0.05$, the heterogeneity was considered to be significant. We combined all of the values from each individual study using the fixed-effect model and the random-effect model. When $P>0.05$, the effects were assumed to be homogenous, and the fixed-effect model (the Mantel-Haenszel method) was used [21]. When $P<0.05$, the random-effect model (the DerSimonian and Laird method) was more appropriate [22]. The inter-study variance $\mathrm{I}^{2}\left(\mathrm{I}^{2}=100 \% \times\right.$ (Q-df)/Q) was used to quantitatively estimate the heterogeneity, and the percentage of $\mathrm{I}^{2}$ was used to describe the extent of the heterogeneity, $\mathrm{I}^{2}<25 \%, 25-75 \%$ and $>75 \%$ represent low, moderate and high inconsistency, respectively $[23,24]$. In addition, we performed sensitivity analyses to evaluate the potential biases of the results in our metaanalyses. The Hardy-Weinberg equilibrium (HWE) of the controls for each study was also calculated using a goodness-of-fit test (chi-square or Fisher's exact test) and $P<0.05$ was considered to be statistically significant. Sensitivity analyses were carried out to assess the stability of the results by conducting analysis of studies with controls in HWE. Finally, the Begg's funnel plot and Egger's test were utilised to estimate the publication bias [25]. All analyses were conducted by the software Stata (Version 11; Stata Corporation, College Station, Texas, USA). All $P$-values were two-sided and a $P$ of $<0.05$ was considered to be statistically significant.

\section{Results}

\section{Studies selected}

Through the literature search and selection, a total of 40 eligible studies met the inclusion criteria and were included in our meta-analysis. One study (Konac et al.) [26] provided data on three types of cancer (cervical cancer, ovarian cancer, and endometrial cancer) and both polymorphisms; therefore, we have grouped them as one in the meta-analyses of all subjects except when stratified by cancer type. Thus, each type of cancer in this study was treated as a separated study in sub-group analyses. Among the 40 eligible studies, 40 studies, representing 10869 cases and 14289 controls, were ultimately analyzed for the HIF-1 $\alpha$ C1772T polymorphism [8,17,26-63], and 30 studies, representing 7177 cases and 10442 controls, were analyzed for the
HIF-1 $\alpha$ G1790A polymorphism [8,17,26,29-31,33-35,37-43, 45-48,50,52-57,59,62,63]. The literature search and study selection procedure are shown in Figure 1. Of the 40 studies on the HIF-1 $\alpha$ C1772T polymorphism, 6 studies were conducted on prostate cancer, 6 studies on breast cancer, 3 studies on lung cancer, 4 studies on colorectal cancer, 4 studies on renal cancer, 4 studies on oral cancer and 12 studies on other cancers. Among these eligible studies, 20 were studies on Asians, 16 were studies on Caucasians and 4 studies were performed on a population of mixed ethnicity. The control sources were population-based in 17 studies and hospital-based in 23 studies. For the HIF- $1 \alpha$ G1790A polymorphism, 15 of the 30 eligible studies were performed in Asian populations, 13 studies were performed in Caucasian populations and 2 studies were performed in a mixed ethnicity population. Of these studies, 4 studies were conducted on breast cancer, 3 studies on lung cancer, 4 studies on oral cancer, 3 studies on prostate cancer, 3 studies on cervical cancer, 2 studies on pancreatic cancer, 2 studies on colorectal cancer, 4 studies on renal cancer and 7 studies on other cancers. The control sources were population-based in 17 studies and hospitalbased in 13 studies. The genotype frequency data of the HIF- $1 \alpha$ C1772T and HIF-1 $\alpha$ G1790A polymorphisms were extracted from all of these eligible publications. For the HIF-1 $\alpha$ C1772T polymorphism, the distributions of the genotypes in the control groups in 11 studies were not in HWE [17,50,51,53,54,56-58,60-62]. For the HIF-1 $\alpha$ G1790A polymorphism there was 1 study not in HWE [62]. The main characteristics of the eligible studies in the meta-analysis are listed in Table 1.

\section{Quantitative data synthesis}

For the HIF-1 $\alpha$ C1772T polymorphism, the overall results from the eligible studies demonstrated a significant association between the HIF-1 $\alpha \mathrm{C} 1772 \mathrm{~T}$ polymorphism and an increased cancer risk in four genetic models (TT vs. CC: $\mathrm{OR}=1.63,95 \% \mathrm{CI}=1.02-2.60$; CT $+\mathrm{TT}$ vs. CC: $\mathrm{OR}=1.15$, 95\% CI =1.01-1.34; TT vs. CT + CC: $\mathrm{OR}=2.11,95 \% \mathrm{CI}=$ 1.32-3.77; $\mathrm{T}$ vs. $\mathrm{C}: \mathrm{OR}=1.21,95 \% \mathrm{CI}=1.04-1.41)$. In the subgroup analysis by cancer type, the HIF-1 $\alpha$ C1772T polymorphism significantly increased the risk of breast cancer in Asians (TT vs. CC: $\mathrm{OR}=4.42,95 \% \mathrm{CI}=1.60$ 12.21; TT vs. CT + CC: $\mathrm{OR}=4.16,95 \% \mathrm{CI}=1.51-11.48 ; \mathrm{T}$ vs. $\mathrm{C}$ : $\mathrm{OR}=1.28,95 \% \mathrm{CI}=1.05-1.55)$, other cancers (TT vs. $\mathrm{CC}: \mathrm{OR}=3.18,95 \% \mathrm{CI}=1.90-5.32 ; \mathrm{TT}$ vs. $\mathrm{CT}+\mathrm{CC}$ : $\mathrm{OR}=3.31,95 \% \mathrm{CI}=1.98-5.53$; $\mathrm{T}$ vs. $\mathrm{C}: \mathrm{OR}=1.47,95 \% \mathrm{CI}=$ 1.10-1.96) and lung cancer (TT vs. CT $+\mathrm{CC}: \mathrm{OR}=3.27$, 95\% CI $=1.73-6.17)$. When the data was stratified by ethnicity, the HIF-1 $\alpha$ C1772T polymorphism was significantly correlated with an increased cancer risk in Asian population ( $\mathrm{TT}$ vs. $\mathrm{CC}$ : $\mathrm{OR}=4.10,95 \% \mathrm{CI}=2.49$ 6.76; CT + TT vs. CC: $\mathrm{OR}=1.29,95 \% \quad \mathrm{CI}=1.04-1.58$; TT vs. $\mathrm{CT}+\mathrm{CC}: \mathrm{OR}=3.67,95 \% \mathrm{CI}=2.23-6.02 ; \mathrm{T}$ vs. 


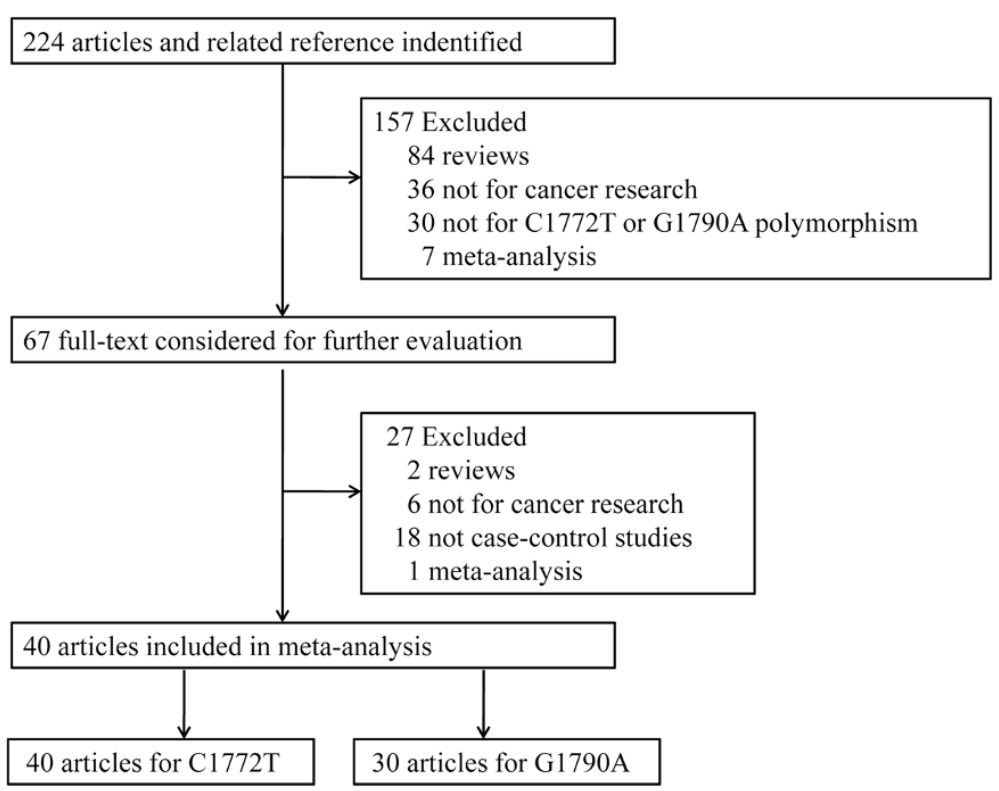

Figure 1 Study flow-chart illustrating the literature search and eligible study selection process.

$\mathrm{C}: \mathrm{OR}=1.28,95 \% \mathrm{CI}=1.04-1.57)$ and Caucasian population (TT vs. CT + CC: $\mathrm{OR}=1.95,95 \% \mathrm{CI}=1.14-3.31$ ). In the analysis stratified by the sources of controls, a significant association was observed in the hospital-based group $(\mathrm{CT}+\mathrm{TT}$ vs. $\mathrm{CC}: \mathrm{OR}=1.28,95 \% \mathrm{CI}=1.01-1.62 ; \mathrm{T}$ vs. $\mathrm{C}: \mathrm{OR}=1.33,95 \% \mathrm{CI}=1.04-1.71)$ and the population-based group (TT vs. $\mathrm{CT}+\mathrm{CC}: \mathrm{OR}=2.01,95 \% \mathrm{CI}=1.10-3.71$ ). Sensitivity analyses were carried out to assess the stability of the results by conducting analyses of studies with controls in HWE. The results showed significantly increased cancer risk ( $\mathrm{TT}$ vs. $\mathrm{CC}$ : $\mathrm{OR}=2.47,95 \% \mathrm{CI}=1.81-3.36$; $\mathrm{CT}+\mathrm{TT}$ vs. $\mathrm{CC}: \mathrm{OR}=1.25,95 \% \mathrm{CI}=1.05-1.49 ; \mathrm{TT}$ s. $\mathrm{CT}+\mathrm{CC}: \mathrm{OR}=2.43,95 \% \mathrm{CI}=1.41-4.19 ; \mathrm{T}$ vs. $\mathrm{C}: \mathrm{OR}=$ $1.27,95 \% \mathrm{CI}=1.06-1.52)$. The other results for the HIF-1 $\alpha$ C1772T polymorphism were similar to those when the studies with controls not in HWE were included. The main results of this pooled analysis are shown in Table 2. Figure 2 shows the forest plot of the association between cancer risk and the HIF- $1 \alpha$ C1772T polymorphism under the allelic model.

For HIF-1 $\alpha$ G1790A polymorphism, as shown in Table 3, the association between the HIF-1 $\alpha$ G1790A polymorphism and increased cancer risk was significant for the pooled ORs under all of the genetic models (AA vs. GG: OR $=5.11,95 \%$ $\mathrm{CI}=2.08-12.56$; GA vs. GG: $\mathrm{OR}=1.45,95 \% \mathrm{CI}=1.05-1.99$; $\mathrm{AA}+\mathrm{AG}$ vs. $\mathrm{GG}: \mathrm{OR}=1.63,95 \% \mathrm{CI}=1.16-2.30 ; \mathrm{AA} v s$. $\mathrm{GA}+\mathrm{GG}: \mathrm{OR}=4.41,95 \% \mathrm{CI}=1.80-10.84 ; \mathrm{A}$ vs. $\mathrm{G}: \mathrm{OR}=$ $1.77,95 \% \mathrm{CI}=1.23-2.25)$. In the subgroup analysis by cancer type, a significant association was observed in lung cancer (AA vs. GG: OR = 5.42, 95\% CI = 2.74-10.70; GA vs. GG: $\mathrm{OR}=1.72,95 \% \mathrm{CI}=1.22-2.41 ; \mathrm{AA}+\mathrm{AG}$ vs. GG: OR = $2.14,95 \% \mathrm{CI}=1.56-2.94 ; \mathrm{AA}$ vs. $\mathrm{GA}+\mathrm{GG}: \mathrm{OR}=4.52,95 \%$
$\mathrm{CI}=2.31-8.83 ; \quad \mathrm{A}$ vs. $\mathrm{G}: \mathrm{OR}=2.27,95 \% \mathrm{CI}=1.74-2.95)$, pancreatic cancer (AA + AG vs. GG: $\mathrm{OR}=3.14,95 \% \mathrm{CI}=$ 1.99-2.97; A vs. $\mathrm{G}$ : $\mathrm{OR}=3.08,95 \% \mathrm{CI}=1.98-4.78)$ and renal cancer (AA vs. GA + GG: OR=3.09, 95\% CI=1.38-6.92). When the data were stratified by ethnicity, significantly increased cancer risk was observed in Asian population and Caucasian population. When the studies were stratified by the source of controls, a significant association was observed for population-based controls under the homozygote model, the dominant comparison model and the allelic model. Sensitivity analyses were conducted after the removal of the studies with controls not in HWE, the results for the HIF-1 $\alpha$ G1790A polymorphism were similar to those when the studies with controls not in HWE were included. Table 3 shows the main results of this pooled analysis for the HIF-1 $\alpha$ G1790A polymorphism. Figure 3 shows the forest plot of the association between cancer risk and the HIF-1 $\alpha$ G1790A polymorphism under the dominant model.

\section{Test of heterogeneity}

There was significant heterogeneity observed in the allelic comparison model, the dominant comparison model and the heterozygote comparison model (Tables 2 and 3), and the heterogeneity was effectively decreased or removed in the subgroups stratified by ethnicity, cancer types and source of controls (Tables 2 and 3).

\section{Sensitivity analysis}

We performed sensitivity analysis by removing each individual study (including the restudies with controls not in HWE) sequentially for both the HIF-1 $\alpha$ C1772T and 
Table 1 Characteristics of studies included in the meta-analysis

\begin{tabular}{|c|c|c|c|c|c|c|c|c|c|c|c|c|c|c|c|}
\hline \multirow{2}{*}{$\begin{array}{l}\text { First } \\
\text { author }\end{array}$} & \multirow[t]{2}{*}{ Year } & \multirow[t]{2}{*}{ Country } & \multirow[t]{2}{*}{ Ethnicity } & \multirow{2}{*}{$\begin{array}{l}\text { Cancer } \\
\text { type }\end{array}$} & \multirow{2}{*}{$\begin{array}{l}\text { Gene } \\
\text { type }\end{array}$} & \multirow{2}{*}{$\begin{array}{l}\text { Source of } \\
\text { controls }\end{array}$} & \multirow{2}{*}{ Cases } & \multicolumn{2}{|c|}{ Controls } & \multicolumn{2}{|l|}{ Case } & \multicolumn{2}{|c|}{ Control } & \multicolumn{2}{|l|}{ HWE } \\
\hline & & & & & & & & & $\mathrm{MM}$ & MW & WW & MM & MW & WW & \\
\hline \multirow[t]{2}{*}{ Clifford } & 2001 & UK & Caucasian & Renal & $\mathrm{C} 1772 \mathrm{~T}$ & PB & 48 & 143 & 42 & 6 & 0 & 110 & 27 & 6 & 0.02 \\
\hline & & & & & G1790A & PB & 48 & 144 & 47 & 1 & 0 & 140 & 4 & 0 & 0.87 \\
\hline \multirow[t]{2}{*}{ Tanimoto } & 2003 & Japan & Asian & HNSCC & $\mathrm{C} 1772 \mathrm{~T}$ & PB & 55 & 110 & 45 & 10 & 0 & 98 & 12 & 0 & 0.55 \\
\hline & & & & & G1790A & PB & 55 & 110 & 51 & 4 & 0 & 101 & 9 & 0 & 0.65 \\
\hline Kuwai & 2004 & Japan & Asian & Colorectal & $\mathrm{C} 1772 \mathrm{~T}$ & PB & 100 & 100 & 100 & 0 & 0 & 89 & 11 & 0 & 0.56 \\
\hline \multirow[t]{2}{*}{ Ollerenshaw } & 2004 & UK & Caucasian & Renal & C1772T & PB & 160 & 162 & 16 & 54 & 90 & 1 & 90 & 71 & 0.001 \\
\hline & & & & & G1790A & PB & 146 & 288 & 65 & 67 & 14 & 239 & 39 & 10 & 0.001 \\
\hline Ling & 2005 & China & Asian & Esophageal & $\mathrm{C} 1772 \mathrm{~T}$ & $\mathrm{HB}$ & 95 & 104 & 84 & 11 & 0 & 93 & 11 & 0 & 0.57 \\
\hline Chau & 2005 & USA & Caucasian & Prostate & $\mathrm{C} 1772 \mathrm{~T}$ & PB & 196 & 196 & 161 & 29 & 6 & 179 & 14 & 3 & 0.002 \\
\hline Fransen & 2006 & Sweden & Caucasian & Colorectal & $\mathrm{C} 1772 \mathrm{~T}$ & PB & 198 & 258 & 167 & 28 & 3 & 213 & 43 & 2 & 0.92 \\
\hline Fransen & 2006 & Sweden & Caucasian & Colorectal & G1790A & PB & 198 & 256 & 189 & 9 & 0 & 247 & 9 & 0 & 0.77 \\
\hline \multirow[t]{6}{*}{ Konac } & 2007 & Turkey & Caucasian & Cervical & $\mathrm{C} 1772 \mathrm{~T}$ & $H B$ & 32 & 107 & 10 & 14 & 8 & 68 & 37 & 2 & 0.23 \\
\hline & & & & & G1790A & $\mathrm{HB}$ & 32 & 107 & 32 & 0 & 0 & 107 & 0 & 0 & 0.99 \\
\hline & & & Caucasian & Ovarian & $\mathrm{C} 1772 \mathrm{~T}$ & $\mathrm{HB}$ & 49 & 107 & 34 & 14 & 1 & 68 & 37 & 2 & 0.23 \\
\hline & & & & & G1790A & $\mathrm{HB}$ & 49 & 107 & 47 & 2 & 0 & 107 & 0 & 0 & 0.99 \\
\hline & & & Caucasian & Endometrial & C1772T & $\mathrm{HB}$ & 21 & 107 & 4 & 12 & 5 & 68 & 37 & 2 & 0.23 \\
\hline & & & & & G1790A & $\mathrm{HB}$ & 21 & 107 & 21 & 0 & 0 & 107 & 0 & 0 & 0.99 \\
\hline \multirow[t]{2}{*}{ Li } & 2007 & USA & mixed & Prostate & $\mathrm{C} 1772 \mathrm{~T}$ & PB & 1041 & 1234 & 818 & 209 & 14 & 995 & 221 & 18 & 0.16 \\
\hline & & & & & G1790A & PB & 1066 & 1264 & 1053 & 13 & 0 & 1247 & 17 & 0 & 0.81 \\
\hline \multirow[t]{2}{*}{ Orr-Urtreger } & 2007 & Israel & Caucasian & Prostate & $\mathrm{C} 1772 \mathrm{~T}$ & PB & 402 & 300 & 287 & 99 & 16 & 217 & 80 & 3 & 0.14 \\
\hline & & & & & G1790A & PB & 200 & 300 & 198 & 2 & 0 & 298 & 2 & 0 & 0.95 \\
\hline \multirow[t]{2}{*}{ Apaydin } & 2008 & Turkey & Caucasian & Breast & $\mathrm{C} 1772 \mathrm{~T}$ & PB & 102 & 102 & 79 & 21 & 2 & 68 & 29 & 5 & 0.42 \\
\hline & & & & & G1790A & PB & 102 & 102 & 102 & 0 & 0 & 98 & 4 & 0 & 0.84 \\
\hline Lee & 2008 & Korea & Asian & Breast & $\mathrm{C} 1772 \mathrm{~T}$ & PB & 1332 & 1369 & 1207 & 119 & 6 & 1245 & 123 & 1 & 0.25 \\
\hline \multirow[t]{2}{*}{ Kim } & 2008 & Korea & Asian & Breast & $\mathrm{C} 1772 \mathrm{~T}$ & $H B$ & 90 & 102 & 81 & 8 & 1 & 93 & 9 & 0 & 0.64 \\
\hline & & & & & G1790A & $H B$ & 90 & 102 & 87 & 3 & 0 & 94 & 7 & 1 & 0.06 \\
\hline \multirow[t]{2}{*}{ Nadaoka } & 2008 & Japan & Asian & Bladder & C1772T & $\mathrm{HB}$ & 219 & 461 & 197 & 21 & 1 & 419 & 42 & 0 & 0.35 \\
\hline & & & & & G1790A & $\mathrm{HB}$ & 219 & 461 & 204 & 13 & 2 & 421 & $40^{*}$ & - & 0.46 \\
\hline Jacobs & 2008 & USA & mixed & Prostate & $\mathrm{C} 1772 \mathrm{~T}$ & $H B$ & 1420 & 1450 & 1156 & 252 & 12 & 1138 & 284 & 28 & 0.04 \\
\hline Horree & 2008 & Netherland & Caucasian & Endometrial & C1772T & PB & 58 & 559 & 50 & 5 & 3 & 463 & 84 & 12 & 0.01 \\
\hline Naidu & 2009 & Malaysia & Asian & Breast & $\mathrm{C} 1772 \mathrm{~T}$ & PB & 410 & 275 & 294 & 100 & 16 & 222 & 50 & 3 & 0.92 \\
\hline & & & & & G1790A & PB & 410 & 275 & 332 & 72 & 6 & 232 & 41 & 2 & 0.90 \\
\hline Chen & 2009 & Taiwan & Asian & Oral & $\mathrm{C} 1772 \mathrm{~T}$ & PB & 174 & 347 & 163 & 10 & 1 & 334 & 13 & 0 & 0.72 \\
\hline & & & & & G1790A & PB & 174 & 347 & 153 & 20 & 1 & 333 & 14 & 0 & 0.70 \\
\hline Konac & 2009 & Turkey & Caucasian & Lung & $\mathrm{C} 1772 \mathrm{~T}$ & PB & 141 & 156 & 110 & 31 & 0 & 111 & 43 & 2 & 0.34 \\
\hline & & & & & G1790A & PB & 141 & 156 & 141 & 1 & 0 & 154 & 2 & 0 & 0.94 \\
\hline Morris & 2009 & UK & Caucasian & Renal & $\mathrm{C} 1772 \mathrm{~T}$ & PB & 332 & 313 & 290 & 39 & 3 & 262 & 46 & 5 & 0.08 \\
\hline & & & & & G1790A & PB & 325 & 309 & 313 & 10 & 2 & 294 & 15 & 0 & 0.66 \\
\hline Foley & 2009 & Ireland & Caucasian & Prostate & $\mathrm{C} 1772 \mathrm{~T}$ & PB & 95 & 188 & 65 & 30 & 0 & 175 & 13 & 0 & 0.62 \\
\hline
\end{tabular}


Table 1 Characteristics of studies included in the meta-analysis (Continued)

\begin{tabular}{|c|c|c|c|c|c|c|c|c|c|c|c|c|c|c|c|}
\hline \multirow[t]{2}{*}{$\mathrm{Li}$} & 2009 & China & Asian & Gastric & C1772T & $\mathrm{HB}$ & 87 & 106 & 83 & 4 & 0 & 93 & 13 & 0 & 0.50 \\
\hline & & & & & G1790A & $\mathrm{HB}$ & 87 & 106 & 74 & 13 & 0 & 100 & 6 & 0 & 0.76 \\
\hline \multicolumn{16}{|l|}{ Munoz- } \\
\hline \multirow[t]{2}{*}{ Guerra } & 2009 & Spain & Caucasian & Oral & C1772T & PB & 70 & 148 & 57 & 6 & 7 & 113 & 27 & 8 & $<0.01$ \\
\hline & & & & & G1790A & PB & 64 & 139 & 40 & 21 & 3 & 130 & 9 & 0 & 0.69 \\
\hline \multirow[t]{2}{*}{ Kim } & 2010 & Korea & Asian & Cervical & C1772T & $\mathrm{HB}$ & 199 & 214 & 177 & 22 & 0 & 187 & 27 & 0 & 0.32 \\
\hline & & & & & G1790A & $\mathrm{HB}$ & 199 & 213 & 187 & 12 & 0 & 200 & 12 & 1 & 0.10 \\
\hline \multirow[t]{2}{*}{ Shieh } & 2010 & Taiwan & Asian & Oral & C1772T & $\mathrm{HB}$ & 305 & 96 & 282 & 23 & 0 & 89 & 7 & 0 & 0.71 \\
\hline & & & & & G1790A & $\mathrm{HB}$ & 305 & 96 & 281 & 24 & 0 & 89 & 7 & 0 & 0.71 \\
\hline \multirow[t]{2}{*}{ Knechtal } & 2010 & Austria & Caucasian & Colorectal & C1772T & PB & 368 & 2156 & 291 & $77^{* *}$ & - & 1773 & $383^{*}$ & - & $>0.05$ \\
\hline & & & & & G1790A & PB & 367 & 2156 & 356 & $11^{*}$ & - & 2080 & $76^{*}$ & - & $>0.05$ \\
\hline \multirow[t]{2}{*}{ Hsiao } & 2010 & Taiwan & Asian & $\begin{array}{l}\text { Hepatocellul- } \\
\text { ar }\end{array}$ & $\mathrm{C} 1772 \mathrm{~T}$ & $\mathrm{HB}$ & 102 & 347 & 94 & 8 & 0 & 334 & 13 & 0 & 0.72 \\
\hline & & & & & G1790A & $\mathrm{HB}$ & 102 & 347 & 87 & 15 & 0 & 333 & 14 & 0 & 0.70 \\
\hline Xu & 2011 & China & Asian & Glioma & C1772T & $\mathrm{HB}$ & 150 & 150 & 121 & 27 & 2 & 135 & 14 & 1 & 0.35 \\
\hline \multirow[t]{2}{*}{ Putra } & 2011 & Japan & Asian & Lung & C1772T & PB & 83 & 110 & 74 & 9 & 0 & 98 & 12 & 0 & 0.55 \\
\hline & & & & & G1790A & PB & 83 & 110 & 72 & 9 & 2 & 101 & 9 & 0 & 0.65 \\
\hline Kang & 2011 & Korea & Asian & Colorectal & $\mathrm{C} 1772 \mathrm{~T}$ & PB & 50 & 50 & 46 & $4^{* *}$ & - & 38 & $12^{* *}$ & - & $<0.01$ \\
\hline \multirow[t]{2}{*}{ Wang } & 2011 & China & Asian & Pancreatic & C1772T & $\mathrm{HB}$ & 263 & 271 & 209 & 54 & 0 & 242 & 29 & 0 & 0.35 \\
\hline & & & & & G1790A & $\mathrm{HB}$ & 263 & 271 & 198 & 65 & 0 & 249 & 22 & 0 & 0.49 \\
\hline Zagouri & 2012 & Greece & Caucasian & Breast & C1772T & $\mathrm{HB}$ & 113 & 124 & 98 & 15 & 0 & 107 & 17 & 0 & 0.41 \\
\hline \multirow[t]{2}{*}{ Kuo } & 2012 & Taiwan & Asian & Lung & C1772T & $\mathrm{HB}$ & 285 & 300 & 153 & 94 & 38 & 216 & 73 & 11 & 0.13 \\
\hline & & & & & G1790A & $\mathrm{HB}$ & 285 & 300 & 150 & 94 & 41 & 215 & 74 & 11 & 0.15 \\
\hline \multirow[t]{2}{*}{ Qin } & 2012 & China & Asian & Renal & C1772T & $\mathrm{HB}$ & 620 & 623 & 572 & 46 & 2 & 578 & 43 & 2 & 0.22 \\
\hline & & & & & G1790A & $\mathrm{HB}$ & 620 & 623 & 575 & 45 & 0 & 584 & 39 & 0 & 0.42 \\
\hline \multirow[t]{2}{*}{ Li } & 2012 & China & Asian & Prostate & C1772T & $\mathrm{HB}$ & 662 & 716 & 612 & 48 & 2 & 659 & 57 & 0 & 0.27 \\
\hline & & & & & G1790A & $\mathrm{HB}$ & 662 & 716 & 614 & 47 & 1 & 685 & 31 & 0 & 0.55 \\
\hline \multirow[t]{2}{*}{ Alves } & 2012 & Brazil & mixed & Oral & C1772T & PB & 40 & 88 & 0 & 1 & 39 & 0 & 85 & 3 & $<0.01$ \\
\hline & & & & & G1790A & PB & 40 & 88 & 2 & 1 & 37 & 81 & 7 & 0 & 0.70 \\
\hline \multirow[t]{2}{*}{ Ruiz-Tovar } & 2012 & Spain & Caucasian & Pancreatic & C1772T & PB & 59 & 152 & 47 & 1 & 11 & 116 & 28 & 8 & 0.002 \\
\hline & & & & & G1790A & PB & 59 & 152 & 54 & 2 & 3 & 142 & 10 & 0 & 0.68 \\
\hline \multirow[t]{2}{*}{$\mathrm{Fu}$} & 2013 & China & Asian & Cervical & $\mathrm{C} 1772 \mathrm{~T}$ & $\mathrm{HB}$ & 518 & 553 & 467 & 49 & 2 & 492 & 60 & 1 & 0.55 \\
\hline & & & & & G1790A & $\mathrm{HB}$ & 509 & 553 & 489 & 20 & 0 & 510 & 42 & 1 & 0.89 \\
\hline \multirow[t]{2}{*}{ Ribeiro } & 2013 & Portugal & Caucasian & Breast & $\mathrm{C} 1772 \mathrm{~T}$ & PB & 96 & 74 & 74 & 21 & 1 & 61 & 7 & 4 & 0.001 \\
\hline & & & & & G1790A & PB & 96 & 74 & 96 & 0 & 0 & 74 & 0 & 0 & 0.99 \\
\hline \multicolumn{16}{|l|}{ Mera- } \\
\hline Menendez & 2013 & Spain & Caucasian & Glottic & & & & & & & & & & & \\
\hline \multirow[t]{2}{*}{ larynx } & C1772T & $\mathrm{HB}$ & 118 & 148 & 85 & 18 & 15 & 113 & 27 & 8 & 0.001 & & & & \\
\hline & & & & & G1790A & $\mathrm{HB}$ & 111 & 139 & 107 & 4 & 0 & 130 & 9 & 0 & 0.69 \\
\hline \multirow[t]{2}{*}{ Total } & & & & & C1772T & & 10869 & 14289 & 8994 & 1568 & 307 & 12181 & 1897 & 211 & \\
\hline & & & & & G1790A & & 7117 & 10442 & 6416 & 589 & 112 & 9922 & 494 & 26 & \\
\hline
\end{tabular}


Table 2 Meta-analysis of the HIF-1a C1772T polymorphism and cancer risk

\begin{tabular}{|c|c|c|c|c|c|c|c|c|c|c|c|c|c|c|c|c|c|c|c|c|c|}
\hline \multirow[t]{2}{*}{ Variables } & \multirow[b]{2}{*}{ Study } & \multirow[b]{2}{*}{$\begin{array}{l}\text { Casel } \\
\text { control }\end{array}$} & \multicolumn{4}{|c|}{ TT vs. CC } & \multicolumn{4}{|c|}{ CT vs. CC } & \multicolumn{4}{|c|}{$\mathrm{CT}+\mathrm{TT}$ vs. $\mathrm{CC}$} & \multicolumn{4}{|c|}{ TT vs. $\mathrm{CT}+\mathrm{CC}$} & \multicolumn{3}{|c|}{ T vs. C } \\
\hline & & & $I^{2}$ & Phet & OR $(95 \% \mathrm{Cl})$ & $\begin{array}{l}\text { Casel } \\
\text { control }\end{array}$ & $I^{2}$ & Phet & OR $(95 \% \mathrm{Cl})$ & $\begin{array}{l}\text { Casel } \\
\text { control }\end{array}$ & $P^{2}$ & Phet & OR $(95 \% \mathrm{Cl})$ & $\begin{array}{l}\text { case/ } \\
\text { control }\end{array}$ & $I^{2}$ & Phet & OR $(95 \% \mathrm{Cl})$ & $\begin{array}{l}\text { Casel } \\
\text { control }\end{array}$ & $\overline{r^{2}}$ & Phet & OR $(95 \%$ Cl) \\
\hline Overall & 40 & $\begin{array}{l}9301 / \\
12392\end{array}$ & 67 & $<0.001$ & $1.63(1.02-2.60)^{*}$ & $\begin{array}{l}10562 / \\
14078\end{array}$ & 68 & $<0.001$ & $1.08(0.92-1.26)^{*}$ & $\begin{array}{l}10958 / \\
14676\end{array}$ & 70 & $<0.001$ & $1.15(1.01-1.34)^{*}$ & $\begin{array}{l}10540 / \\
12470\end{array}$ & 71 & $<0.001$ & $2.11(1.32-3.37)^{*}$ & $\begin{array}{l}21738 / \\
28578\end{array}$ & 76 & $<0.001$ & $1.21(1.04-1.41)^{*}$ \\
\hline $\begin{array}{l}\text { Overall } \\
\text { in HWE }\end{array}$ & 31 & $\begin{array}{l}7429 / \\
9947\end{array}$ & 59 & 0.02 & $2.21(1.27-3.83)^{*}$ & $\begin{array}{l}8481 / \\
11109\end{array}$ & 64 & $<0.001$ & $1.15(0.98-1.36)^{*}$ & $\begin{array}{l}8604 / \\
11556\end{array}$ & 70 & $<0.001$ & $1.20(1.02-1.41)^{*}$ & $\begin{array}{l}8275 / \\
9350\end{array}$ & 49 & 0.01 & $2.13(1.28-3.55)^{*}$ & $\begin{array}{l}17208 / \\
22338\end{array}$ & 76 & $<0.001$ & $1.22(1.03-1.44)^{*}$ \\
\hline \multicolumn{22}{|l|}{$\begin{array}{l}\text { Cancer } \\
\text { type }\end{array}$} \\
\hline Cervical & 3 & $\begin{array}{l}664 / \\
750\end{array}$ & 66 & 0.09 & $10.11(2.55-40.05)$ & $\begin{array}{l}739 / \\
871\end{array}$ & 60 & 0.08 & $0.98(0.72-1.34)$ & $\begin{array}{l}749 / \\
874\end{array}$ & 80 & 0.01 & $1.32(0.61-2.87)^{*}$ & $\begin{array}{l}749 / \\
874\end{array}$ & 51 & 0.15 & $8.55(2.28-32.13)$ & $\begin{array}{l}2369 / \\
1748\end{array}$ & 88 & $<0.001$ & $1.41(0.59-3.35)^{*}$ \\
\hline Breast & 6 & $\begin{array}{l}1859 / \\
1809\end{array}$ & 62 & 0.03 & $1.41(0.34-5.75)^{*}$ & $\begin{array}{l}2117 / \\
2033\end{array}$ & 37 & 0.16 & $1.01(0.91-1.33)$ & $\begin{array}{l}2143 / \\
2046\end{array}$ & 46 & 0.1 & $1.13(0.94-1.36)$ & $\begin{array}{l}2143 / \\
2046\end{array}$ & 60 & 0.04 & $1.38(0.35-5.46)^{*}$ & $\begin{array}{l}4286 / \\
4092\end{array}$ & 56 & 0.04 & $1.09(0.80-1.48)^{*}$ \\
\hline $\begin{array}{l}\text { Breast } \\
\text { in HWE }\end{array}$ & 5 & $\begin{array}{l}1784 / \\
1744\end{array}$ & 55 & 0.08 & $2.30(1.08-4.91)$ & $\begin{array}{l}2022 / \\
1963\end{array}$ & 35 & 0.19 & $1.07(0.88-1.29)$ & $\begin{array}{l}2047 / \\
1972\end{array}$ & 56 & 0.06 & $1.12(0.92-1.35)$ & $\begin{array}{l}2047 / \\
1972\end{array}$ & 49 & 0.12 & $2.27(1.06-4.82)$ & $\begin{array}{l}4154 / \\
3944\end{array}$ & 65 & 0.02 & $1.09(0.76-1.55)^{*}$ \\
\hline $\begin{array}{l}\text { Breast } \\
\text { in Asian }\end{array}$ & 3 & $\begin{array}{l}1605 / \\
1564\end{array}$ & 0 & 0.93 & $4.42(1.60-12.21)$ & $\begin{array}{l}1809 / \\
1742\end{array}$ & 36 & 0.21 & $1.14(0.92-1.41)$ & $\begin{array}{l}1832 / \\
1746\end{array}$ & 51 & 0.13 & $1.22(0.99-1.49)$ & $\begin{array}{l}1832 / \\
1746\end{array}$ & 0 & 0.91 & $4.16(1.51-11.48)$ & $\begin{array}{l}3664 / \\
3492\end{array}$ & 55 & 0.11 & $1.28(1.05-1.55)$ \\
\hline Lung & 3 & $\begin{array}{l}375 / \\
438\end{array}$ & 75 & 0.04 & $1.41(0.07-30.44)^{*}$ & $\begin{array}{l}471 / \\
553\end{array}$ & 75 & 0.02 & $1.13(0.59-2.19)^{*}$ & $\begin{array}{l}509 / \\
566\end{array}$ & 86 & 0.01 & $1.19(0.51-2.76)^{*}$ & $\begin{array}{l}509 / \\
566\end{array}$ & 71 & 0.07 & $3.27(1.73-6.17)$ & $\begin{array}{l}1018 / \\
1132\end{array}$ & 89 & $<0.001$ & $1.19(0.50-2.86)^{*}$ \\
\hline Colorectal & 4 & $\begin{array}{l}599 / \\
2123\end{array}$ & - & - & - & $\begin{array}{l}624 / \\
2175\end{array}$ & 79 & 0.03 & $0.24(0.01-5.51)^{*}$ & $\begin{array}{l}627 / \\
2177\end{array}$ & 71 & 0.02 & $1.12(0.57-2.18)^{*}$ & $\begin{array}{l}627 / \\
2177\end{array}$ & - & - & - & $\begin{array}{l}1254 / \\
4354\end{array}$ & 80 & 0.02 & $0.26(0.01-6.38)^{*}$ \\
\hline Prostate & 6 & $\begin{array}{l}3149 / \\
3415\end{array}$ & 70 & 0.01 & $1.34(0.54-3.31)^{*}$ & $\begin{array}{l}3766 / \\
4032\end{array}$ & 86 & $<0.001$ & $1.34(0.93-1.92)^{*}$ & $\begin{array}{l}3816 / \\
4084\end{array}$ & 87 & $<0.001$ & $1.36(0.95-1.96)^{*}$ & $\begin{array}{l}3816 / \\
4084\end{array}$ & 69 & 0.01 & $1.31(0.54-3.20)^{*}$ & $\begin{array}{l}7632 / \\
8168\end{array}$ & 87 & $<0.001$ & $1.35(0.96-1.89)^{*}$ \\
\hline $\begin{array}{l}\text { Prostate } \\
\text { in HWE }\end{array}$ & 4 & $\begin{array}{l}1814 / \\
2067\end{array}$ & 59 & 0.09 & $1.57(0.89-2.75)$ & $\begin{array}{l}2168 / \\
2417\end{array}$ & 88 & $<0.001$ & $1.42(0.84-2.40)^{*}$ & $\begin{array}{l}2200 / \\
2438\end{array}$ & 87 & 0.01 & $1.50(0.89-2.40)^{*}$ & $\begin{array}{l}2200 / \\
2438\end{array}$ & 61 & 0.08 & $1.55(0.89-2.72)$ & $\begin{array}{l}4400 / \\
4876\end{array}$ & 85 & $<0.001$ & $1.44(0.93-2.21)^{*}$ \\
\hline Renal & 4 & $\begin{array}{l}1015 / \\
1035\end{array}$ & 25 & 0.26 & $0.28(0.12-1.28)$ & $\begin{array}{l}1065 / \\
1157\end{array}$ & 74 & 0.01 & $0.62(0.31-1.24)^{*}$ & $\begin{array}{l}1160 / \\
1241\end{array}$ & 70 & 0.02 & $0.62(0.33-1.18)^{*}$ & $\begin{array}{l}1160 / \\
1241\end{array}$ & 21 & 0.29 & $1.37(0.92-2.04)$ & $\begin{array}{l}2320 / \\
2482\end{array}$ & 44 & 0.15 & $0.91(0.73-1.12)$ \\
\hline $\begin{array}{l}\text { Renal in } \\
\text { HWE }\end{array}$ & 2 & $\begin{array}{l}867 / \\
847\end{array}$ & 0 & 0.62 & $0.67(0.21-2.13)$ & $\begin{array}{l}947 / \\
929\end{array}$ & 13 & 0.28 & $0.92(0.67-1.26)$ & $\begin{array}{l}952 / \\
936\end{array}$ & 29 & 0.24 & $0.90(0.67-1.22)$ & $\begin{array}{l}952 / \\
936\end{array}$ & 0 & 0.64 & $0.69(0.22-2.17)$ & $\begin{array}{l}1904 / \\
1872\end{array}$ & 37 & 0.21 & $0.89(0.67-1.19)$ \\
\hline Oral & 4 & $\begin{array}{l}549 / \\
547\end{array}$ & 0 & 0.46 & $2.01(0.75-5.41)$ & $\begin{array}{l}542 / \\
668\end{array}$ & 50 & 0.14 & $0.90(0.55-1.47)$ & $\begin{array}{l}589 / \\
679\end{array}$ & 16 & 0.3 & $1.04(0.66-1.64)$ & $\begin{array}{l}589 / \\
679\end{array}$ & 93 & $<0.001$ & $22.82(0.28-1887.72)^{*}$ & $\begin{array}{l}1178 / \\
1358\end{array}$ & 88 & $<0.001$ & $2.52(0.71-8.98)^{*}$ \\
\hline $\begin{array}{l}\text { Oral in } \\
\text { HWE }\end{array}$ & 2 & $\begin{array}{l}446 / \\
423\end{array}$ & - & - & - & $\begin{array}{l}478 / \\
443\end{array}$ & 0 & 0.5 & $1.28(0.69-2.38)$ & $\begin{array}{l}479 / \\
443\end{array}$ & 0 & 0.4 & $1.35(0.73-2.49)$ & $\begin{array}{l}479 / \\
443\end{array}$ & - & - & - & $\begin{array}{l}958 / \\
886\end{array}$ & 0 & 0.32 & $1.41(0.78-2.56)$ \\
\hline Other & 12 & $\begin{array}{l}1033 / \\
2151\end{array}$ & 30 & 0.2 & $3.18(1.90-5.32)$ & $\begin{array}{l}1190 / \\
2445\end{array}$ & 67 & $<0.001$ & $1.18(0.79-1.78)^{*}$ & $\begin{array}{l}1276 / \\
2622\end{array}$ & 60 & $<0.001$ & $1.34(0.95-1.87)^{*}$ & $\begin{array}{l}1276 / \\
2622\end{array}$ & 0 & 0.52 & $3.31(1.98-5.53)$ & $\begin{array}{l}2434 / \\
4940\end{array}$ & 58 & 0.01 & $1.47(1.10-1.96)^{*}$ \\
\hline $\begin{array}{l}\text { Other in } \\
\text { HWE }\end{array}$ & 9 & $\begin{array}{l}880 / \\
1032\end{array}$ & 56 & 0.08 & $5.10(1.72-15.07)$ & $\begin{array}{l}1032 / \\
1758\end{array}$ & 60 & 0.01 & $1.47(0.97-2.21)^{*}$ & $\begin{array}{l}1041 / \\
1763\end{array}$ & 64 & 0.01 & $1.52(0.99-2.34)^{*}$ & $\begin{array}{l}1041 / \\
1763\end{array}$ & 24 & 0.27 & $4.47(1.53-13.00)$ & $\begin{array}{l}2082 / \\
3526\end{array}$ & 67 & 0.01 & $1.52(1.02-2.28)^{*}$ \\
\hline \multicolumn{22}{|l|}{ Ethnicity } \\
\hline Asian & 20 & $\begin{array}{l}5124 / \\
5781\end{array}$ & 0 & 0.96 & $4.10(2.49-6.76)$ & $\begin{array}{l}5678 / \\
6335\end{array}$ & 50 & 0.01 & $1.20(0.99-1.46)^{*}$ & $\begin{array}{l}5787 / \\
6400\end{array}$ & 75 & $<0.001$ & $1.29(1.04-1.58)^{*}$ & $\begin{array}{l}5787 / \\
6400\end{array}$ & 0 & 0.98 & $3.67(2.23-6.02)$ & $\begin{array}{l}11574 / \\
12800\end{array}$ & 61 & $<0.001$ & $1.28(1.04-1.57)$ \\
\hline Caucasian & 16 & $\begin{array}{l}1791 / \\
4247\end{array}$ & 74 & $<0.001$ & $1.54(0.72-3.27)^{*}$ & $\begin{array}{l}2220 / \\
4781\end{array}$ & 76 & $<0.001$ & $0.93(0.65-1.33)^{*}$ & $\begin{array}{l}2385 / \\
4921\end{array}$ & 59 & 0.01 & $1.07(0.80-1.43)^{*}$ & $\begin{array}{l}2385 / \\
4921\end{array}$ & 58 & 0.003 & $1.95(1.14-3.31)^{*}$ & $\begin{array}{l}4770 / \\
9842\end{array}$ & 78 & $<0.001$ & $1.20(0.91-1.57)$ \\
\hline $\begin{array}{l}\text { Caucasian } \\
\text { in } \mathrm{HWE}\end{array}$ & 9 & $\begin{array}{l}1473 / \\
3153\end{array}$ & 76 & $<0.001$ & $2.28(0.62-8.35)^{*}$ & $\begin{array}{l}1738 / \\
3152\end{array}$ & 79 & $<0.001$ & $1.20(0.99-1.46)^{*}$ & $\begin{array}{l}1776 / \\
3535\end{array}$ & 82 & $<0.001$ & $1.28(0.88-1.86)^{*}$ & $\begin{array}{l}1776 / \\
3535\end{array}$ & 69 & 0.002 & $2.08(0.68-6.37)^{*}$ & $\begin{array}{l}3552 / \\
7070\end{array}$ & 86 & $<0.001$ & $1.34(0.86-2.07)$ \\
\hline
\end{tabular}


Table 2 Meta-analysis of the HIF-1a C1772T polymorphism and cancer risk (Continued)

\begin{tabular}{|c|c|c|c|c|c|c|c|c|c|c|c|c|c|c|c|c|c|c|c|c|c|}
\hline \multicolumn{22}{|c|}{ Source of control } \\
\hline $\mathrm{HB}$ & 17 & $\begin{array}{l}4608 / \\
5249\end{array}$ & 77 & $<0.001$ & $3.28(1.29-8.30)^{*}$ & $\begin{array}{l}5259 / \\
6029\end{array}$ & 60 & $<0.001$ & $1.18(0.96-1.45)^{*}$ & $\begin{array}{l}5348 / \\
6086\end{array}$ & 72 & $<0.001$ & $1.28(1.01-1.62)^{*}$ & $\begin{array}{l}5348 / \\
6086\end{array}$ & 71 & $<0.001$ & $2.85(1.24-6.54)^{*}$ & $\begin{array}{l}10696 / \\
12172\end{array}$ & 80 & $<0.001$ & $1.33(1.04-1.71)^{*}$ \\
\hline $\begin{array}{l}\text { HB in } \\
\text { HWE }\end{array}$ & 15 & $\begin{array}{l}3340 / \\
3962\end{array}$ & 35 & 0.13 & $4.88(2.96-8.04)$ & $\begin{array}{l}3748 / \\
4467\end{array}$ & 56 & 0.01 & $1.24(0.99-1.57)^{*}$ & $\begin{array}{l}3810 / \\
4488\end{array}$ & 67 & $<0.001$ & $1.33(1.02-1.74)^{*}$ & $\begin{array}{l}3810 / \\
4488\end{array}$ & 4 & 0.4 & $4.23(2.58-6.93)$ & $\begin{array}{l}7620 / \\
8976\end{array}$ & 74 & $<0.001$ & $1.38(1.06-1.80)^{*}$ \\
\hline PB & 23 & $\begin{array}{l}4693 / \\
5303\end{array}$ & 54 & 0.01 & $1.33(0.76-2.31)^{*}$ & $\begin{array}{l}5303 / \\
7143\end{array}$ & 74 & $<0.001$ & $0.99(0.77-1.29)^{*}$ & $\begin{array}{l}5521 / \\
8203\end{array}$ & 70 & $<0.001$ & $1.10(0.89-1.36)^{*}$ & $\begin{array}{l}5521 / \\
8203\end{array}$ & 72 & $<0.001$ & $2.02(1.10-3.71)^{*}$ & $\begin{array}{l}11042 / \\
16406\end{array}$ & 74 & $<0.001$ & $1.18(0.95-1.45)^{*}$ \\
\hline $\begin{array}{l}\text { PB in } \\
\text { HWE }\end{array}$ & 15 & $\begin{array}{l}4089 / \\
5985\end{array}$ & 49 & 0.04 & $1.51(0.74-3.11)^{*}$ & $\begin{array}{l}4733 / \\
6642\end{array}$ & 72 & $<0.001$ & $1.10(0.85-1.43)^{*}$ & $\begin{array}{l}4794 / \\
6681\end{array}$ & 72 & $<0.001$ & $1.17(0.93-1.48)^{*}$ & $\begin{array}{l}4794 / \\
6681\end{array}$ & 46 & 0.63 & $1.51(1.01-2.27)$ & $\begin{array}{l}9588 / \\
13362\end{array}$ & 75 & $<0.001$ & $1.14(0.89-1.45)^{\circ}$ \\
\hline
\end{tabular}

HWE: Hardy-Weinberg Equilibrium; PB: population based; HB: hospital based; Phet: P value for heterogeneity. "Random-effects model was used when P value for heterogeneity test <0.05; otherwise, fixed-effects model was used. 
the HIF-1 $\alpha$ G1790A polymorphism (Figure 4 and Additional file 1). The results indicated that the overall significance of the pooled ORs was not altered by any single study in the genetic models for the HIF- $1 \alpha$ C1772T/G1790A polymorphisms and cancer susceptibility, suggesting stability and reliability in our overall results.

\section{Bias diagnostics}

A Begg's funnel plot and Egger's test were used to assess the publication bias in this meta-analysis. As shown in Figure 5, for the HIF-1 $\alpha$ C1772T polymorphism, the funnel plots for the comparison of the five models appear to be basically symmetric. The Egger's linear regression test did not show any evidence of significant publication bias in five models (TT vs. CC: $\mathrm{t}=0.50$, $P=0.62 ;$ TC vs. CC: $\mathrm{t}=-0.19, P=0.85 ;$ TT vs. CT $+\mathrm{CC}$ : $\mathrm{t}=1.11, P=0.28 ; \mathrm{T}$ vs. $\mathrm{C}: \mathrm{t}=1.39, P=0.17 ; \mathrm{CT}+\mathrm{TT} v s$. $\mathrm{CC}: \mathrm{t}=0.59, P=0.56)$. For the HIF-1 $\alpha$ G1790A polymorphism, no visual publication bias was detected in the funnel plot (Figure 6) and the result showed no significant evidence of a publication bias in five models (AA vs. GG: $\mathrm{t}=0.03, P=0.98 ;$ GA $v s . \mathrm{GG}: \mathrm{t}=-0.86$, $P=0.40 ;$ AA $v s . \mathrm{GA}+\mathrm{GG}: \mathrm{t}=0.33, P=0.75 ; \mathrm{AA}+\mathrm{AG} v s$. GG: $\mathrm{t}=-0.40, P=0.69 ; \mathrm{A} v s . \mathrm{G}: \mathrm{t}=-0.41, P=0.68)$.

\section{Discussion}

HIF-1 is a heterodimeric transcription factor and a key regulator of the cellular response to hypoxia [5]. It is composed of HIF- $1 \alpha$ and HIF-1 $\beta$ subunits, which are members of the bHLH-PAS transcription factor family. HIF- $1 \alpha$ is a unique $\mathrm{O}_{2}$-regulated subunit that determines the function of HIF-1. HIF-1 $\alpha$ upregulates the expression of genes whose protein products function to increase $\mathrm{O}_{2}$ availability or to allow metabolic adaptation to $\mathrm{O}_{2}$ deprivation, including VEGF, Epo, NOS2 and others. Most of these aforementioned proteins have been implicated in tumor development and progression $[35,64,65]$. Recent studies have reported that the overexpression of HIF- $1 \alpha$ is significantly associated with cell proliferation, tumor susceptibility, tumor size, lymph node metastasis and prognosis $[12,35,66]$. The HIF-1 $\alpha$ gene is located on 
Table 3 Meta-analysis of the HIF-1a G1790A polymorphism and cancer risk

\begin{tabular}{|c|c|c|c|c|c|c|c|c|c|c|c|c|c|c|c|c|c|c|c|c|c|}
\hline \multirow[t]{2}{*}{ Variables } & \multirow[b]{2}{*}{ Study } & \multirow[b]{2}{*}{$\begin{array}{l}\text { Case/ } \\
\text { control }\end{array}$} & \multicolumn{4}{|c|}{ AA vs. GG } & \multicolumn{4}{|c|}{ GA vs. GG } & \multicolumn{4}{|c|}{$A A+A G$ vs.GG } & \multicolumn{4}{|c|}{$A A$ vs.GA + GG } & \multicolumn{3}{|c|}{ A vs. $G$} \\
\hline & & & $\overline{P^{2}}$ & Phet & OR $(95 \% \mathrm{Cl})$ & $\begin{array}{l}\text { Casel } \\
\text { control }\end{array}$ & $P^{2}$ & Phet & OR $(95 \% \mathrm{Cl})$ & $\begin{array}{l}\text { Casel } \\
\text { control }\end{array}$ & $\overline{P^{2}}$ & Phet & OR $(95 \% \mathrm{Cl})$ & $\begin{array}{l}\text { Casel } \\
\text { control }\end{array}$ & $\overline{I^{2}}$ & Phet & OR $(95 \% \mathrm{Cl})$ & $\begin{array}{l}\text { Case/ } \\
\text { control }\end{array}$ & $\overline{P^{2}}$ & Phet & OR $(95 \% \mathrm{Cl})$ \\
\hline Overall & 30 & $\begin{array}{l}6538 / \\
9948\end{array}$ & 57 & 0.01 & $5.11(2.08-12.56)^{*}$ & $\begin{array}{l}7005 / \\
10442\end{array}$ & 77 & $<0.001$ & $1.45(1.05-1.99)^{*}$ & $\begin{array}{l}7117 / \\
10442\end{array}$ & 83 & $<0.001$ & $1.63(1.16-2.30)^{*}$ & $\begin{array}{l}7117 / \\
10442\end{array}$ & 58 & 0.01 & $4.41(1.80-10.84)^{*}$ & $\begin{array}{l}14234 / \\
20884\end{array}$ & 86 & $<0.001$ & $1.77(1.23-2.25)^{*}$ \\
\hline $\begin{array}{l}\text { Overall } \\
\text { in HWE }\end{array}$ & 29 & $\begin{array}{l}6449 / \\
9699\end{array}$ & 61 & 0.003 & $5.14(1.67-15.86)^{*}$ & $\begin{array}{l}6873 / \\
10138\end{array}$ & 69 & $<0.001$ & $1.35(1.01-1.81)^{*}$ & $\begin{array}{l}6971 / \\
10154\end{array}$ & 79 & $<0.001$ & $1.53(1.10-2.12)^{*}$ & $\begin{array}{l}6971 / \\
10154\end{array}$ & 60 & 0.004 & $4.80(1.58-14.55)^{*}$ & $\begin{array}{l}13942 / \\
20308\end{array}$ & 85 & $<0.001$ & $1.70(1.17-2.46)^{*}$ \\
\hline \multicolumn{22}{|l|}{$\begin{array}{l}\text { Cancer } \\
\text { type }\end{array}$} \\
\hline Breast & 4 & $\begin{array}{l}623 / \\
501\end{array}$ & 0 & 0.34 & $1.44(0.38-5.44)$ & $\begin{array}{l}692 / \\
550\end{array}$ & 53 & 0.12 & $1.03(0.70-1.52)$ & $\begin{array}{l}698 / \\
553\end{array}$ & 60 & 0.08 & $1.05(0.72-1.53)$ & $\begin{array}{l}698 / \\
553\end{array}$ & 0 & 0.36 & $1.41(0.37-5.40)$ & $\begin{array}{l}1396 / \\
1466\end{array}$ & 65 & 0.56 & $1.07(0.76-1.52)$ \\
\hline Cervical & 3 & $\begin{array}{l}708 / \\
819\end{array}$ & 0 & 0.99 & $0.35(0.04-3.39)$ & $\begin{array}{l}740 / \\
871\end{array}$ & 57 & 0.13 & $0.62(0.40-0.98)$ & $\begin{array}{l}740 / \\
837\end{array}$ & 51 & 0.15 & $0.60(0.38-0.94)$ & $\begin{array}{l}740 / \\
837\end{array}$ & 0 & 0.99 & $0.36(0.04-3.450$ & $\begin{array}{l}1480 / \\
1746\end{array}$ & 42 & 0.19 & $0.59(0.38-0.91)$ \\
\hline Oral & 4 & $\begin{array}{l}517 / \\
633\end{array}$ & 75 & 0.02 & $72.11(2.08-2502.44)^{*}$ & $\begin{array}{l}542 / \\
670\end{array}$ & 70 & 0.02 & $3.17(1.26-7.92)^{*}$ & $\begin{array}{l}583 / \\
670\end{array}$ & 92 & $<0.001$ & $7.92(1.58-39.64)^{*}$ & $\begin{array}{l}583 / \\
670\end{array}$ & 75 & 0.02 & $58.05(1.70-1985.77)^{*}$ & $\begin{array}{l}1166 / \\
1340\end{array}$ & 96 & 0.01 & $9.66(1.31-71.15)^{\circ}$ \\
\hline Prostate & 3 & $\begin{array}{l}1866 / \\
2230\end{array}$ & - & - & - & $\begin{array}{l}1927 / \\
2280\end{array}$ & 1 & 0.37 & $1.42(0.97-2.07)$ & $\begin{array}{l}1928 / \\
2280\end{array}$ & 7 & 0.34 & $1.44(0.98-2.10)$ & $\begin{array}{l}1928 / \\
2280\end{array}$ & - & - & - & $\begin{array}{l}3856 / \\
4560\end{array}$ & 10 & 0.33 & $1.45(0.99-2.11)$ \\
\hline Renal & 4 & $\begin{array}{l}1016 / \\
1267\end{array}$ & 0 & 0.95 & $5.10(2.21-11.73)$ & $\begin{array}{l}1123 / \\
1354\end{array}$ & 92 & $<0.001$ & $1.51(0.45-5.05)^{*}$ & $\begin{array}{l}1139 / \\
1364\end{array}$ & 92 & $<0.001$ & $1.58(0.49-5.04)^{*}$ & $\begin{array}{l}1139 / \\
1364\end{array}$ & 0 & 0.76 & $3.09(1.38-6.92)$ & $\begin{array}{l}2278 / \\
2728\end{array}$ & 89 & $<0.001$ & $1.53(0.60-3.92)^{*}$ \\
\hline $\begin{array}{l}\text { Renal } \\
\text { in HWE }\end{array}$ & 3 & $\begin{array}{l}937 / \\
1018\end{array}$ & - & - & - & $\begin{array}{l}991 / \\
1076\end{array}$ & 0 & 0.42 & $1.00(0.69-1.47)$ & $\begin{array}{l}993 / \\
1076\end{array}$ & 0 & 0.6 & $1.04(0.71-1.52)$ & $\begin{array}{l}993 / \\
1076\end{array}$ & - & - & - & $\begin{array}{l}1986 / \\
2152\end{array}$ & 0 & 0.78 & $1.07(0.74-1.55)$ \\
\hline Lung & 3 & $\begin{array}{l}405 / \\
481\end{array}$ & 0 & 0.87 & $5.42(2.74-10.70)$ & $\begin{array}{l}466 / \\
555\end{array}$ & 0 & 0.57 & $1.72(1.22-2.41)$ & $\begin{array}{l}509 / \\
566\end{array}$ & 0 & 0.46 & $2.14(1.56-2.94)$ & $\begin{array}{l}509 / \\
566\end{array}$ & 0 & 0.79 & $4.52(2.31-8.83)$ & $\begin{array}{l}1018 / \\
1132\end{array}$ & 0 & 0.48 & $2.27(1.74-2.95)$ \\
\hline Colorectal & 2 & $\begin{array}{l}545 / \\
2327\end{array}$ & - & - & - & $\begin{array}{l}554 / \\
2336\end{array}$ & - & - & - & $\begin{array}{l}554 / \\
2336\end{array}$ & 0 & 0.45 & $0.97(0.57-1.63)$ & $\begin{array}{l}554 / \\
2336\end{array}$ & - & - & - & $\begin{array}{l}1108 / \\
4672\end{array}$ & - & - & - \\
\hline Pancreatic & 2 & $\begin{array}{l}255 / \\
391\end{array}$ & - & - & - & $\begin{array}{l}319 / \\
423\end{array}$ & 82 & 0.02 & $1.61(0.24-10.76)^{*}$ & $\begin{array}{l}3221 \\
423\end{array}$ & 63 & 0.1 & $3.14(1.99-4.97)$ & $\begin{array}{l}3221 \\
423\end{array}$ & - & - & - & $\begin{array}{l}644 / \\
846\end{array}$ & 0 & 0.42 & $3.08(1.98-4.78)$ \\
\hline Other & 7 & $\begin{array}{l}593 / \\
1377\end{array}$ & - & - & - & $\begin{array}{l}642 / \\
1377\end{array}$ & 74 & $<0.001$ & $1.53(0.65-3.59)^{*}$ & $\begin{array}{l}644 / \\
1377\end{array}$ & 72 & $<0.001$ & $1.57(0.70-3.53)^{*}$ & $\begin{array}{l}644 / \\
1377\end{array}$ & - & - & - & $\begin{array}{l}1288 / \\
2754\end{array}$ & 69 & 0.01 & $1.57(0.75-3.30)^{*}$ \\
\hline \multicolumn{22}{|l|}{ Ethnicity } \\
\hline Asian & 15 & $\begin{array}{l}3607 / \\
4263\end{array}$ & 13 & 0.33 & $3.50(2.05-5.98)$ & $\begin{array}{l}4010 / \\
4614\end{array}$ & 74 & $<0.001$ & $1.44(1.04-1.99)^{*}$ & $\begin{array}{l}4063 / \\
4630\end{array}$ & 76 & $<0.001$ & $1.49(1.07-2.08)^{*}$ & $\begin{array}{l}4063 / \\
4630\end{array}$ & 0 & 0.45 & $3.12(1.83-5.32)$ & $\begin{array}{l}8126 / \\
9260\end{array}$ & 77 & $<0.001$ & $1.49(1.08-2.05)^{*}$ \\
\hline Caucasian & 13 & $\begin{array}{l}1829 / \\
4357\end{array}$ & 0 & 0.69 & $6.63(3.11-14.12)$ & $\begin{array}{l}1926 / \\
4450\end{array}$ & 81 & $<0.001$ & $1.36(0.58-3.19)^{*}$ & $\begin{array}{l}1948 / \\
4460\end{array}$ & 82 & $<0.001$ & $1.45(0.69-3.04)^{*}$ & $\begin{array}{l}1948 / \\
4460\end{array}$ & 0 & 0.49 & $4.21(2.04-8.71)$ & $\begin{array}{l}3896 / \\
8920\end{array}$ & 75 & $<0.001$ & $1.65(0.84-3.24)^{*}$ \\
\hline $\begin{array}{l}\text { Caucasian } \\
\text { in HWE }\end{array}$ & 12 & $\begin{array}{l}1750 / \\
4108\end{array}$ & 0 & 0.74 & $12.40(2.19-70.22)$ & $\begin{array}{l}1794 / \\
4172\end{array}$ & 68 & 0.01 & $1.10(0.48-2.49)^{*}$ & $\begin{array}{l}1802 / \\
4172\end{array}$ & 67 & 0.01 & $1.22(0.62-2.37)^{*}$ & $\begin{array}{l}1802 / \\
4172\end{array}$ & 0 & 0.79 & $11.37(2.02-63.93)$ & $\begin{array}{l}3604 / \\
8344\end{array}$ & 68 & 0.01 & $1.65(1.17-2.32)^{*}$ \\
\hline \multicolumn{22}{|c|}{ Source of control } \\
\hline $\mathrm{HB}$ & 13 & $\begin{array}{l}3197 / \\
3945\end{array}$ & 45 & 0.12 & $1.54(0.35-6.70)$ & $\begin{array}{l}3510 / \\
4234\end{array}$ & 77 & $<0.001$ & $1.37(0.92-2.05)^{*}$ & $\begin{array}{l}3554 / \\
4248\end{array}$ & 79 & $<0.001$ & $1.40(0.93-2.11)^{*}$ & $\begin{array}{l}3554 / \\
4248\end{array}$ & 35 & 0.19 & $3.13(1.74-5.62)$ & $\begin{array}{l}7108 / \\
8496\end{array}$ & 79 & $<0.001$ & $1.38(0.93-2.05)^{*}$ \\
\hline PB & 17 & $\begin{array}{l}3133 / \\
5705\end{array}$ & 66 & 0.01 & $11.55(6.62-20.12)^{*}$ & $\begin{array}{l}3295 / \\
5882\end{array}$ & 78 & $<0.001$ & $1.51(0.88-2.58)^{*}$ & $\begin{array}{l}3563 / \\
6194\end{array}$ & 85 & $<0.001$ & $1.90(1.06-3.39)^{*}$ & $\begin{array}{l}3563 / \\
6194\end{array}$ & 69 & 0.002 & $10.27(2.42-43.63)^{*}$ & $\begin{array}{l}6726 / \\
11788\end{array}$ & 89 & $<0.001$ & $2.25(1.18-4.29)^{*}$ \\
\hline $\begin{array}{l}\text { PB in } \\
\text { HWE }\end{array}$ & 16 & $\begin{array}{l}3054 / \\
5456\end{array}$ & 67 & 0.006 & $15.51(2.53-94.94)^{*}$ & $\begin{array}{l}3163 / \\
5604\end{array}$ & 60 & 0.01 & $1.34(0.85-2.11)^{*}$ & $\begin{array}{l}3417 / \\
5906\end{array}$ & 81 & $<0.001$ & $1.71(0.97-3.03)^{*}$ & $\begin{array}{l}3417 / \\
5906\end{array}$ & 66 & 0.007 & $14.20(2.38-84.61)^{*}$ & $\begin{array}{l}6434 / \\
11212\end{array}$ & 89 & $<0.001$ & $2.33(1.91-2.84)^{*}$ \\
\hline
\end{tabular}


chromosome 14q21-24 and contains a total of 35 common SNPs, according to the dbSNP database (http://www. ncbi.nlm.nih.gov/SNP). Two polymorphisms, C1772T (rs11549465) and G1790A (rs11549467), result in an amino acid substitution of proline to serine and alanine to threonine, respectively, and the present studies show that C1772T (rs11549465) is not in substantial linkage disequilibrium (LD) with G1790A (rs11549467) $\left(R^{2}=0.002\right)$. Under normoxic condition, the hydroxylation of proline 402 and proline 564 occurs within the oxygen-dependent degradation (ODD) domain of HIF- $1 \alpha$, and HIF- $1 \alpha$ is rapidly degraded. The two SNPs examined here are located within the ODD/pVHL binding domain in exon 12 of the HIF- $1 \alpha$ gene and may enhance the transcription activity of the HIF- $1 \alpha$ gene by causing structural changes, increasing the stability of HIF-1 $\alpha$ protein and affecting the expression of downstream target genes $[8,14,17]$. Over the last few years, a great number of studies have been performed to investigate the association between these HIF- $1 \alpha$ polymorphisms and cancer risk in different populations. However, the results of these studies remain inconclusive. In a meta-analysis conducted by Zhao et al. in 2009 [67], the HIF-1 C1772T polymorphism was reported to be associated with increased cancer risk, while no significant association was found between the HIF-1 $\alpha$ G1790A polymorphism and cancer risk. Additionally, Li et al. reported that the HIF-1 $\alpha \mathrm{C} 1772 \mathrm{~T}$ polymorphism correlates with urinary cancer risk in Caucasian population, and the G1790A polymorphism may increase the risk of prostate cancer [68]. Due to the important role of HIF- $1 \alpha$ polymorphisms in the development of cancer and due to the limited statistical power of the previous studies, we conducted a comprehensive literature search and performed a meta-analysis on all of the available case-control studies to systematically evaluate the exact relationship between the C1772T/G1790A polymorphisms in HIF-1 $\alpha$ and cancer susceptibility.

Regarding the HIF-1 $\alpha$ C1772T polymorphism, our results suggested a significant association in four genetic comparison models, providing convincing evidence that the HIF-1 $\alpha$ C1772T polymorphism may be a risk factor in cancer development. When sensitivity analyses were performed, the results were similar to those when the studies with controls not in HWE were included, suggesting that our results were very robust. Moreover, when the data were stratified by cancer type, a significant association was observed between the $\mathrm{C} 1772 \mathrm{~T}$ polymorphism and breast cancer in Asians. This may be due to the specific genetic variant induced over-expression of HIF-1 under hypoxic condition in breast cancer cells, and the different life style, ethnicity and body composition between Asians and Caucasians, which could contribute to the results. A significant association was also observed in lung cancer. When subgroup analyses were performed according to ethnicity and source of controls, a significant association was found in Asian population, Caucasian population and in hospital-based studies. Zhao et al. [67] reported that the genotype TT was significantly associated with an increased cancer risk in Asians, but the CI was very wide due to the lack of mutant homozygotes in Asians. In our meta-analysis, we also found that the C1772T polymorphism was a risk factor in Asians (Dominant model: $\mathrm{OR}=1.29$, 95\% CI $=1.04-1.58$; Allelic model: $\mathrm{OR}=1.47$, $95 \% \mathrm{CI}=1.04-1.57)$. Beyond that, we had not found any significant associations in prostate cancer, renal cancer or oral cancer.

For the HIF-1 $\alpha$ G1790A polymorphism, the pooled results from all of the eligible studies suggested that the G1790A polymorphism in HIF- $1 \alpha$ is significantly associated with an increased cancer risk in all of the genetic models. We also conducted subgroup analyses based on the cancer type, ethnicities and source of controls. In the subgroup analysis according to cancer type, the results suggested that the HIF-1 $\alpha$ G1790A polymorphism significantly increased the risk of lung cancer, renal cancer, oral cancer and pancreatic cancer, but the CI for the oral cancer subgroup was very wide. This may be due to the lack of mutant homozygotes detected, and the association could have been caused by chance. More studies based on large populations should be prusued. The study reported by Putra et al. indicated that even though they did not found any significant differences in genotype for G1790A between lung cancer patients and healthy controls, however, the G1790A variant allele was significantly higher in lung cancer patients, and TP53 LOH and $1 \mathrm{p} 34 \mathrm{LOH}$ were more frequently observed in individuals with the HIF-1 $\alpha$ G1790A polymorphism, suggesting that this polymorphism may induce mutations in some tumor suppressor genes involved in lung cancer development [46]. Here, we found a significant association between the G1790A polymorphism and lung cancer risk. When the data were stratified according to ethnicity classification and source of controls, similar to the $\mathrm{C} 1772 \mathrm{~T}$ polymorphism, significantly increased risks were also found in Asian populations, Caucasian populations and population-based studies. After sensitivity analyses were performed, our results did not vary substantially, which strongly suggests an association between the HIF-1 $\alpha$ G1790A polymorphism and increased cancer risk. One important factor that could influence the results is heterogeneity. In our study, significant heterogeneity existed in the analysis of the heterozygote model, the dominant model and the allelic model for the HIF-1 $\alpha$ C1772T/G1790A polymorphism. When we performed a subgroup analysis according to cancer type, ethnicity or source of controls, the heterogeneity was reduced significantly or disappeared. The significant heterogeneity may due to the differences in ethnicity or 


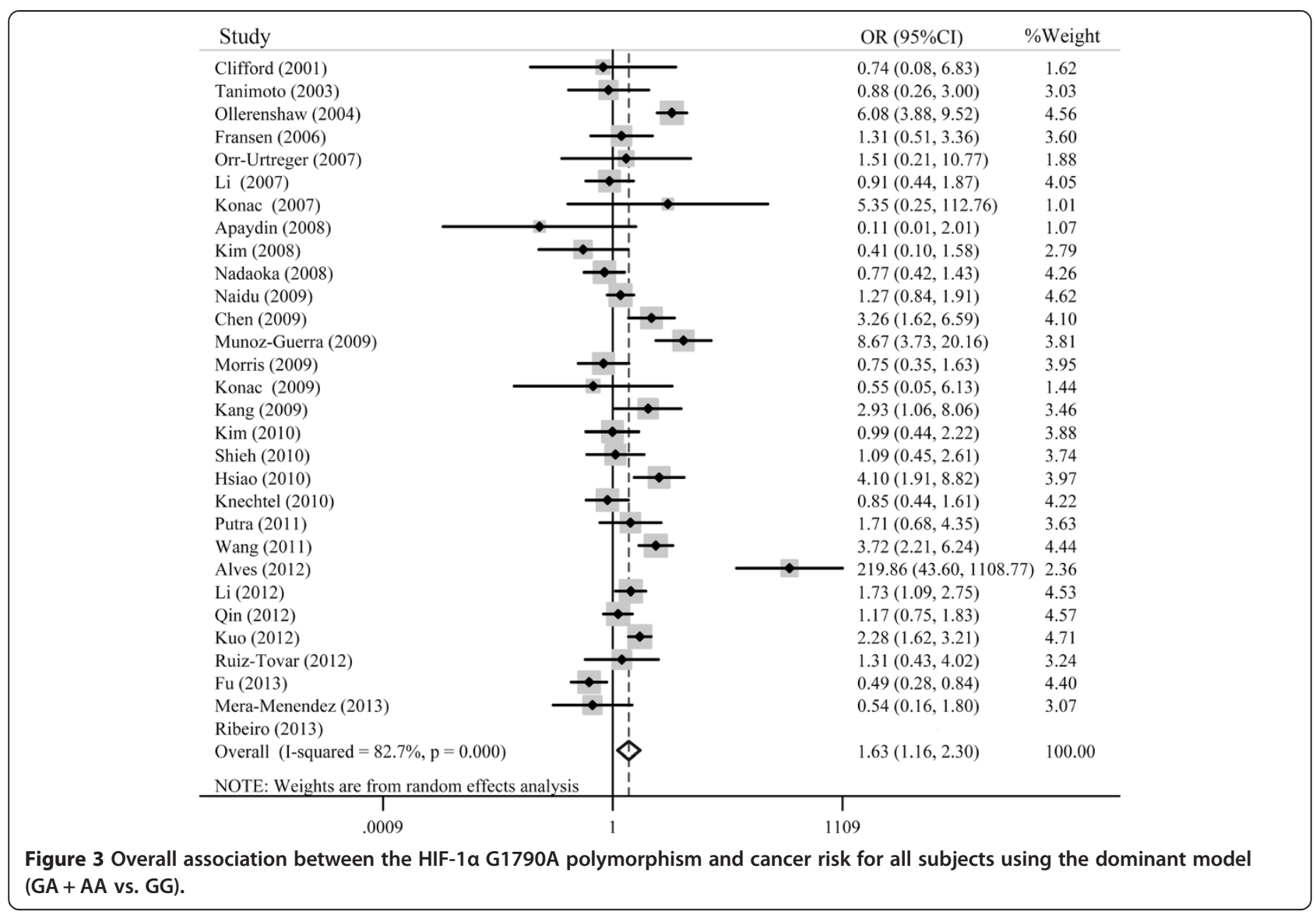

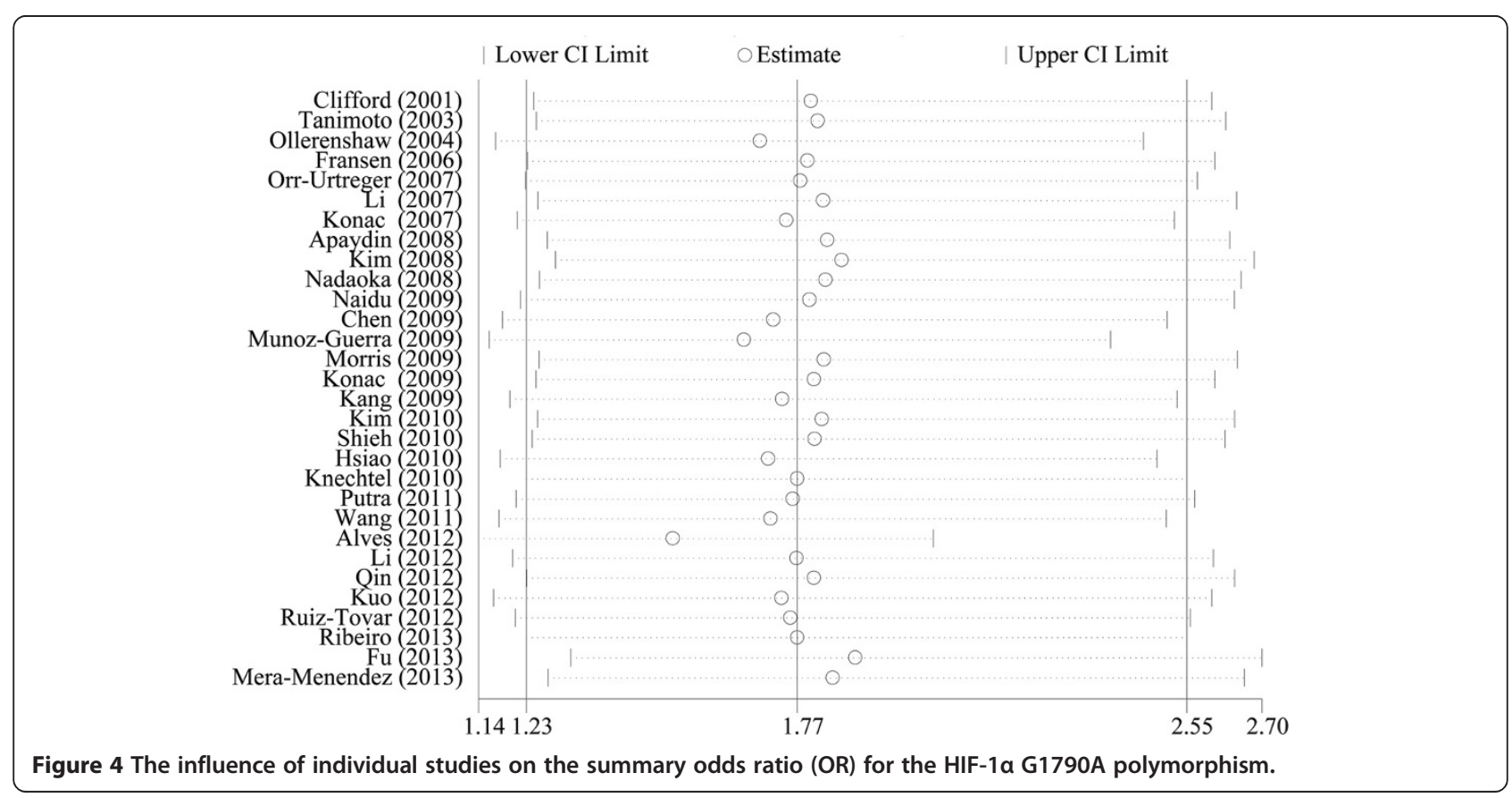




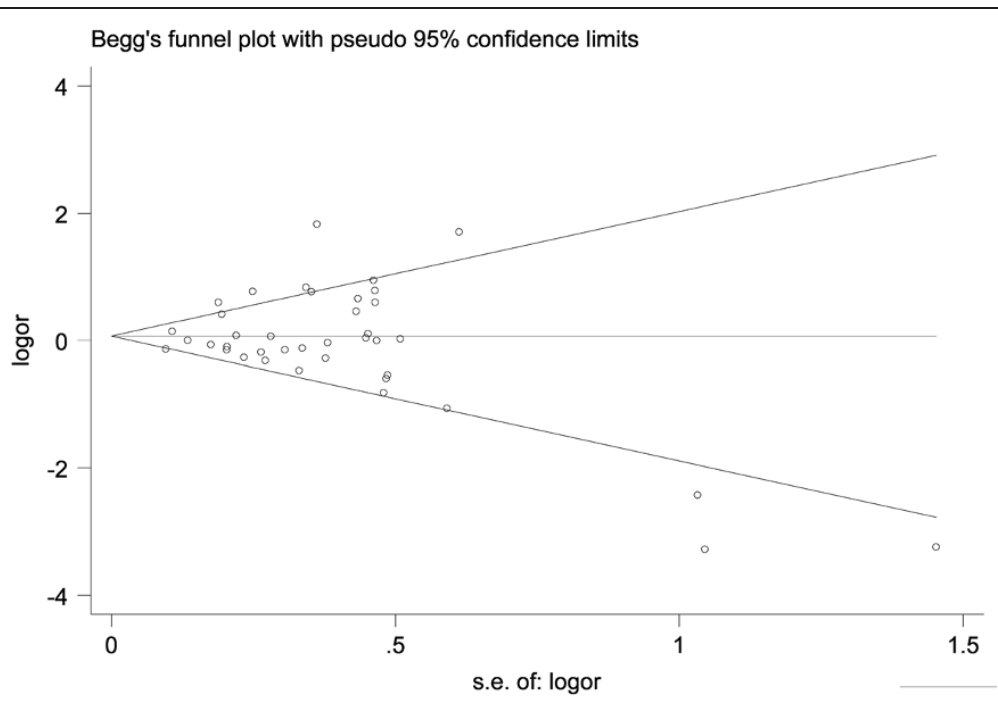

Figure 5 Begg's funnel plot for evaluating the publication bias of the meta-analysis for the HIF-1a C1772T polymorphism.

cancer types or even in the selection of the controls. Furthermore, publication bias was not observed in our meta-analysis of the HIF-1 $\alpha$ G1790A/C1772T polymorphisms. We also performed a sensitivity analysis to evaluate the sources of heterogeneity. The pooled ORs did not vary substantially, indicating that the results of our meta-analysis are robust and reliable.

To a certain extent, our meta-analysis still includes several limitations that should be interpreted and taken into consideration. First, in the era of GWAS, researchers can obtain the GWAS data for these two SNPs from all cancer studies and conduct a meta-analysis with the GWAS data instead of relying on published data, which may be biased toward positive findings. Second, the lack of observations concerning gene-gene and geneenvironment interactions could influence our results. Third, although the total number of studies was not small, there were still not sufficient eligible studies for us to analyze different types of cancers, such as colorectal carcinoma, renal cell carcinoma or glioma; more studies are needed to explore the potential relationship between HIF-1 $\alpha$ C1772T/G1790A polymorphisms and cancer susceptibility. Forth, the lack of detailed original data, such as the age and sex of the populations, smoking

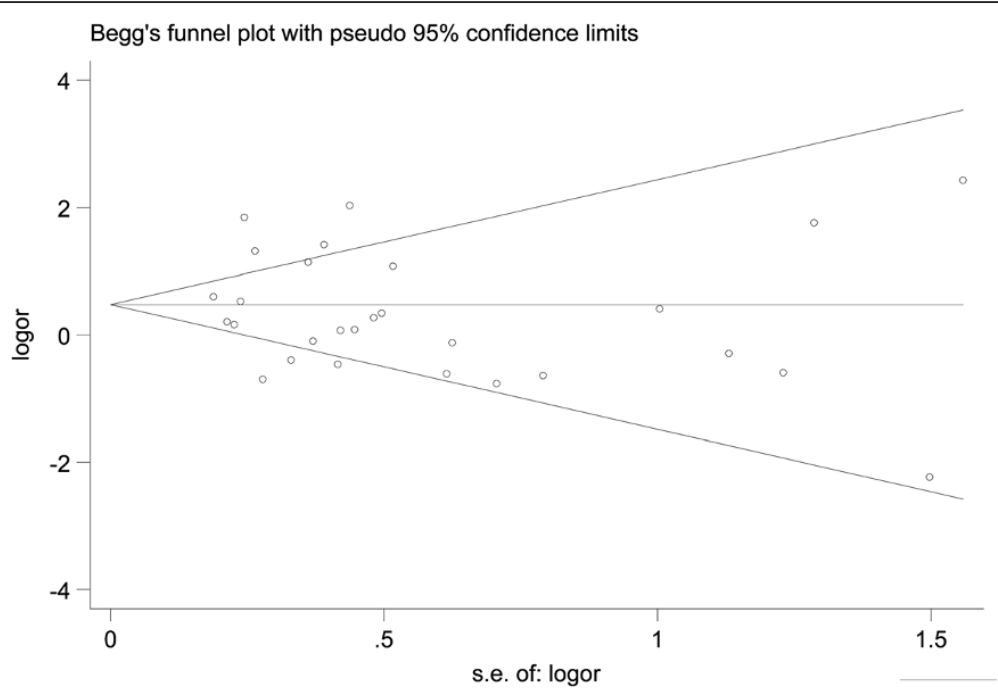

Figure 6 Begg's funnel plot for evaluating the publication bias of the meta-analysis for the HIF-1a G1790A polymorphism. 
status, or alcohol consumption in the eligible studies may influence our extended analyses. However, our metaanalysis also has many advantages. First, we searched all possible publications, and the total number of eligible studies was much larger than other previously published meta-analyses; therefore, our results are more convincing. Second, no publication bias was detected in our metaanalysis. Finally, all of the data were extracted from wellselected studies, providing stronger statistical power for our study.

\section{Conclusions}

In conclusion, this meta-analysis provides powerful evidence that both the $\mathrm{C} 1772 \mathrm{~T}$ and G1790A polymorphisms in the HIF-1 $\alpha$ gene may contribute to individual susceptibility to cancers. It will be necessary to perform additional research to evaluate the relationship between HIF- $1 \alpha$ C1772T/G1790A polymorphisms and cancer risk. Moreover, large sample case-control studies assessing gene-togene and gene-to-environment interactions are required to verify these findings.

\section{Additional file}

Additional file 1: The influence of individual studies on the summary odds ratio (OR) for the HIF-1a C1772T polymorphism.

\section{Competing interests}

The authors declare that they have no competing interests.

\section{Authors' contributions}

QY participated in collection of data and manuscript preparation. PC and QY SW performed the statistical analysis and participated in the revision. PZ and $\mathrm{NL}, \mathrm{AG}$ participated in study design and critically revised the manuscript. PZ participated in study design and manuscript preparation. All authors read and approved the final manuscript.

\section{Authors' information}

Qing Yan, Pin Chen and Songtao Wang are joint first authors.

\section{Acknowledgements}

This work is supported by the National Natural Science Foundation of China (grant 30901534, 81172694 and 81473013); the Grant for the 135 Key Medical Project of Jiangsu Province (No. XK201117); and the National high technology research and development program 863 (No. 2012AA02A508).

Received: 31 March 2013 Accepted: 20 November 2014

Published: 15 December 2014

\section{References}

1. Jemal A, Bray F, Center MM, Ferlay J, Ward E, Forman D: Global cancer statistics. CA Cancer J Clin 2011, 61(2):69-90.

2. Jemal A, Siegel R, Ward E, Hao Y, Xu J, Thun MJ: Cancer statistics, 2009. CA Cancer J Clin 2009, 59(4):225-249.

3. Pharoah PD, Dunning AM, Ponder BA, Easton DF: Association studies for finding cancer-susceptibility genetic variants. Nat Rev Cancer 2004, 4(11):850-860.

4. Hoeijmakers $\mathrm{JH}$ : Genome maintenance mechanisms for preventing cancer. Nature 2001, 411(6835):366-374

5. Wang GL, Jiang BH, Rue EA, Semenza GL: Hypoxia-inducible factor 1 is a basic-helix-loop-helix-PAS heterodimer regulated by cellular $\mathrm{O} 2$ tension. Proc Natl Acad Sci U S A 1995, 92(12):5510-5514.
6. Semenza GL: HIF-1 and mechanisms of hypoxia sensing. Curr Opin Cell Biol 2001, 13(2):167-171.

7. Sutter $\mathrm{CH}$, Laughner $\mathrm{E}$, Semenza GL: Hypoxia-inducible factor 1alpha protein expression is controlled by oxygen-regulated ubiquitination that is disrupted by deletions and missense mutations. Proc Natl Acad Sci U S A 2000, 97(9):4748-4753.

8. Tanimoto K, Yoshiga K, Eguchi H, Kaneyasu M, Ukon K, Kumazaki T, Oue N, Yasui W, Imai K, Nakachi K, Poellinger L, Nishiyama M: Hypoxia-inducible factor-1alpha polymorphisms associated with enhanced transactivation capacity, implying clinical significance. Carcinogenesis 2003, 24(11):1779-1783.

9. Bardos Jl, Ashcroft M: Negative and positive regulation of HIF-1: a complex network. Biochim Biophys Acta 2005, 1755(2):107-120.

10. Ravi R, Mookerjee B, Bhujwalla ZM, Sutter $C H$, Artemov D, Zeng Q, Dillehay LE, Madan A, Semenza GL, Bedi A: Regulation of tumor angiogenesis by $\mathrm{p} 53$-induced degradation of hypoxia-inducible factor 1alpha. Genes Dev 2000, 14(1):34-44.

11. Ryan HE, Poloni M, McNulty W, Elson D, Gassmann M, Arbeit JM, Johnson RS: Hypoxia-inducible factor-1alpha is a positive factor in solid tumor growth. Cancer Res 2000, 60(15):4010-4015.

12. Semenza GL: Targeting HIF-1 for cancer therapy. Nat Rev Cancer 2003, 3(10):721-732

13. Rannala B: Finding genes influencing susceptibility to complex diseases in the post-genome era. Am J Pharmacogenomics 2001, 1(3):203-221.

14. Yamada N, Horikawa Y, Oda N, lizuka K, Shihara N, Kishi S, Takeda J: Genetic variation in the hypoxia-inducible factor-1alpha gene is associated with type 2 diabetes in Japanese. J Clin Endocrinol Metab 2005, 90(10):5841-5847.

15. Semenza GL, Rue EA, lyer NV, Pang MG, Kearns WG: Assignment of the hypoxia-inducible factor 1 alpha gene to a region of conserved synteny on mouse chromosome 12 and human chromosome 14q. Genomics 1996, 34(3):437-439.

16. Fu XS, Choi E, Bubley GJ, Balk SP: Identification of hypoxia-inducible factor-1alpha (HIF-1alpha) polymorphism as a mutation in prostate cancer that prevents normoxia-induced degradation. Prostate 2005, 63(3):215-221.

17. Clifford SC, Astuti D, Hooper L, Maxwell PH, Ratcliffe PJ, Maher ER: The pVHL-associated SCF ubiquitin ligase complex: molecular genetic analysis of elongin $B$ and $C, R b x 1$ and HIF-1alpha in renal cell carcinoma. Oncogene 2001, 20(36):5067-5074.

18. Smaldone MC, Maranchie JK: Clinical implications of hypoxia inducible factor in renal cell carcinoma. Urol Oncol 2009, 27(3):238-245.

19. Yoshiba S, Ito D, Nagumo T, Shirota T, Hatori M, Shintani S: Hypoxia induces resistance to 5 -fluorouracil in oral cancer cells via $G(1)$ phase cell cycle arrest. Oral Oncol 2009, 45(2):109-115.

20. Lin PY, Yu CH, Wang JT, Chen HH, Cheng SJ, Kuo MY, Chiang CP: Expression of hypoxia-inducible factor- 1 alpha is significantly associated with the progression and prognosis of oral squamous cell carcinomas in Taiwan. J Oral Pathol Med 2008, 37(1):18-25.

21. Mantel N, Haenszel W: Statistical aspects of the analysis of data from retrospective studies of disease. J Natl Cancer Inst 1959, 22(4):719-748.

22. DerSimonian R, Laird N: Meta-analysis in clinical trials. Control Clin Trials 1986, 7(3):177-188

23. Higgins JP, Thompson SG: Quantifying heterogeneity in a meta-analysis Stat Med 2002, 21(11):1539-1558.

24. Higgins JP, Thompson SG, Deeks JJ, Altman DG: Measuring inconsistency in meta-analyses. BMJ 2003, 327(7414):557-560.

25. Egger M, Davey Smith G, Schneider M, Minder C: Bias in meta-analysis detected by a simple, graphical test. BMJ 1997, 315(7109):629-634

26. Konac E, Onen HI, Metindir J, Alp E, Biri AA, Ekmekci A: An investigation of relationships between hypoxia-inducible factor-1 alpha gene polymorphisms and ovarian, cervical and endometrial cancers. Cancer Detect Prev 2007, 31(2):102-109.

27. Kuwai T, Kitadai Y, Tanaka S, Kuroda T, Ochiumi T, Matsumura S, Oue N, Yasui W, Kaneyasu M, Tanimoto K, Nishyama M, Chayama K: Single nucleotide polymorphism in the hypoxia-inducible factor-1alpha gene in colorectal carcinoma. Oncol Rep 2004, 12(5):1033-1037.

28. Ling TS, Shi RH, Zhang GX, Zhu H, Yu LZ, Ding XF: Common single nucleotide polymorphism of hypoxia-inducible factor-1alpha and its impact on the clinicopathological features of esophageal squamous cell carcinoma. Chin J Dig Dis 2005, 6(4):155-158. 
29. Fransen K, Fenech M, Fredrikson M, Dabrosin C, Soderkvist P: Association between ulcerative growth and hypoxia inducible factor-1alpha polymorphisms in colorectal cancer patients. Mol Carcinog 2006, 45(11):833-840.

30. Orr-Urtreger A, Bar-Shira A, Matzkin H, Mabjeesh NJ: The homozygous P582S mutation in the oxygen-dependent degradation domain of HIF-1 alpha is associated with increased risk for prostate cancer. Prostate 2007, 67(1):8-13.

31. Li P, Cao Q, Shao PF, Cai HZ, Zhou H, Chen JW, Qin C, Zhang ZD, Ju XB, Yin CJ: Genetic polymorphisms in HIF1A are associated with prostate cancer risk in a Chinese population. Asian J Androl 2012, 14(6):864-869.

32. Lee JY, Choi JY, Lee KM, Park SK, Han SH, Noh DY, Ahn SH, Kim DH, Hong YC, Ha E, Yoo KY, Ambrosone CB, Kang D: Rare variant of hypoxia-inducible factor-1alpha (HIF-1A) and breast cancer risk in Korean women. Clin Chim Acta 2008, 389(1-2):167-170.

33. Kim HO, Jo YH, Lee J, Lee SS, Yoon KS: The C1772T genetic polymorphism in human HIF-1alpha gene associates with expression of HIF-1alpha protein in breast cancer. Oncol Rep 2008, 20(5):1181-1187.

34. Apaydin I, Konac E, Onen HI, Akbaba M, Tekin E, Ekmekci A: Single nucleotide polymorphisms in the hypoxia-inducible factor-1alpha (HIF-1alpha) gene in human sporadic breast cancer. Arch Med Res 2008, 39(3):338-345

35. Chen MK, Chiou HL, Su SC, Chung TT, Tseng HC, Tsai HT, Yang SF: The association between hypoxia inducible factor-1alpha gene polymorphisms and increased susceptibility to oral cancer. Oral Oncol 2009, 45(12):e222-e226.

36. Foley $R$, Marignol L, Thomas AZ, Cullen IM, Perry AS, Tewari P, O'Grady A, Kay E, Dunne B, Loftus B, Waston WR, Fitzpatirck JM, Woodson K, Lehman T, Hollywood D, Lynch TH, Lawler M: The HIF-1alpha C1772T polymorphism may be associated with susceptibility to clinically localised prostate cancer but not with elevated expression of hypoxic biomarkers. Cancer Biol Ther 2009, 8(2):118-124

37. Konac E, Dogan I, Onen HI, Yurdakul AS, Ozturk C, Varol A, Ekmecki A: Genetic variations in the hypoxia-inducible factor-1alpha gene and lung cancer. Exp Biol Med (Maywood) 2009, 234(9):1109-1116.

38. Morris MR, Hughes DJ, Tian YM, Ricketts CJ, Lau KW, Gentle D, Shuib S, Serrano-Fernandez P, Lubinski J, Wiesener MS, Pugh CW, Latif F, Ratcliffe PJ, Maher ER: Mutation analysis of hypoxia-inducible factors HIF1A and HIF2A in renal cell carcinoma. Anticancer Res 2009, 29(11):4337-4343.

39. Li K, Zhang Y, Dan Z, Wang Y, Ren ZC: Association of the hypoxia inducible factor-1alpha gene polymorphisms with gastric cancer in Tibetans. Biochem Genet 2009, 47(9-10):625-634

40. Naidu R, Har YC, Taib NA: Associations between hypoxia-inducible factor-1alpha (HIF-1alpha) gene polymorphisms and risk of developing breast cancer. Neoplasma 2009, 56(5):441-447.

41. Kim YH, Park IA, Park WY, Kim JW, Kim SC, Park NH, Song YS, Kang SB: Hypoxia-inducible factor 1alpha polymorphisms and early-stage cervical cancer. Int J Gynecol Cancer 2011, 21(1):2-7.

42. Hsiao PC, Chen MK, Su SC, Ueng KC, Chen YC, Hsieh YH, Liu YF, Tsai HT, Yang SF: Hypoxia inducible factor-1alpha gene polymorphism G1790A and its interaction with tobacco and alcohol consumptions increase susceptibility to hepatocellular carcinoma. J Surg Oncol 2010, 102(2):163-169.

43. Shieh TM, Chang KW, Tu HF, Shih YH, Ko SY, Chen YC, Liu CJ: Association between the polymorphisms in exon 12 of hypoxia-inducible factor-1alpha and the clinicopathological features of oral squamous cell carcinoma. Oral Oncol 2010, 46(9):e47-e53.

44. Xu G, Wang M, Xie W, Bai X: Hypoxia-inducible factor-1 alpha C1772T gene polymorphism and glioma risk: a hospital-based case-control study from China. Genet Test Mol Biomark 2011, 15(6):461-464.

45. Wang X, Liu Y, Ren H, Yuan Z, Li S, Sheng J, Zhao T, Chen Y, Liu F, Wang F, Huang $\mathrm{H}$, Hao J: Polymorphisms in the hypoxia-inducible factor-1alpha gene confer susceptibility to pancreatic cancer. Cancer Biol Ther 2011, 12(5):383-387.

46. Putra AC, Tanimoto K, Arifin M, Hiyama K: Hypoxia-inducible factor-1alpha polymorphisms are associated with genetic aberrations in lung cancer. Respirology 2011, 16(5):796-802.

47. Kuo WH, Shih CM, Lin CW, Cheng WE, Chen SC, Chen W, Lee YL: Association of hypoxia inducible factor-1alpha polymorphisms with susceptibility to non-small-cell lung cancer. Trans/ Res 2012, 159(1):42-50.
48. Qin C, Cao Q, Ju X, Wang M, Meng X, Zhu J, Yan F, Li P, Ding Q, Chen J, Gu M, Zhang W, Yin C, Zhang Z: The polymorphisms in the VHL and HIF1A genes are associated with the prognosis but not the development of renal cell carcinoma. Ann Oncol 2012, 23(4):981-989.

49. Zagouri F, Sergentanis TN, Gazouli M, Tsigginou A, Dimitrakakis C, Papaspyrou I, Eleutherakis-Papaiakovou E, Chrysikos D, Theodoropoulos G, Zografos GC, Antsaklis A, Dimopoulos AM, Papadimitriou CA: HSP90, HSPA8, HIF-1 alpha and HSP70-2 polymorphisms in breast cancer: a case-control study. Mol Biol Rep 2012, 39(12):10873-10879.

50. Ribeiro AL, Gaspar JF, Pereira T, Ribeiro V: Lack of relevance of HIF-1alpha polymorphisms in breast cancer in a Portuguese population. Anticancer Res 2013, 33(6):2549-2555.

51. Kang MJ, Jung SA, Jung JM, Kim SE, Jung HK, Kim TH, Shim KN, Yi SY, Yoo K, Moon IH: Associations between single nucleotide polymorphisms of MMP2, VEGF, and HIF1A genes and the risk of developing colorectal cancer. Anticancer Res 2011, 31(2):575-584

52. Knechtel G, Szkandera J, Stotz M, Hofmann G, Langsenlehner U, Krippl P, Samonigg H, Renner W, Langner C, Dehchamani D, Gerger A: Single nucleotide polymorphisms in the hypoxia-inducible factor-1 gene and colorectal cancer risk. Mol Carcinog 2010, 49(9):805-809.

53. Alves LR, Fraga CA, Oliveira MV, Sousa AA, Jorge AS, Marques-Silva L, Santos SH, Jones KM, de Paula AM, Guimaraes AL: High HIF-1alpha expression genotypes increase odds ratio of oral cancer. Head Neck Oncol 2012, 4(5):87.

54. Munoz-Guerra MF, Fernandez-Contreras ME, Moreno AL, Martin ID, Herraez B, Gamallo C: Polymorphisms in the hypoxia inducible factor 1-alpha and the impact on the prognosis of early stages of oral cancer. Ann Surg Oncol 2009, 16(8):2351-2358.

55. Fu SL, Miao J, Ding B, Wang XL, Cheng WJ, Dai HH, Han SP: A polymorphism in the $3^{\prime}$ untranslated region of Hypoxia-Inducible Factor-1 alpha confers an increased risk of cervical cancer in aChinese population. Neoplasma 2013, 61(1):63-69.

56. Mera-Menendez F, Hinojar-Gutierrez A, Guijarro Rojas M, de Gregorio JG, Mera-Menendez E, Sanchez JJ, Quintanilla M, Cerezo L, Gamallo C: Polymorphisms in HIF-1alpha affect presence of lymph node metastasis and can influence tumor size in squamous-cell carcinoma of the glottic larynx. Clin Transl Oncol 2013, 15(5):358-363.

57. Ruiz-Tovar J, Fernandez-Contreras ME, Martin-Perez E, Gamallo C: Association of thymidylate synthase and hypoxia inducible factor-1alpha DNA polymorphisms with pancreatic cancer. Tumori 2012, 98(3):364-369.

58. Horree N, Groot AJ, van Hattem WA, Heintz AP, Vooijs M, van Diest PJ: HIF-1A gene mutations associated with higher microvessel density in endometrial carcinomas. Histopathology 2008, 52(5):637-639.

59. Nadaoka J, Horikawa Y, Saito M, Kumazawa T, Inoue T, Narita S, Yuasa T, Satoh S, Nishiyama H, Ogawa O, Tsuchiya N, Habuchi T: Prognostic significance of HIF-1 alpha polymorphisms in transitional cell carcinoma of the bladder. Int J Cancer 2008, 122(6):1297-1302.

60. Jacobs EJ, Hsing AW, Bain EB, Stevens VL, Wang Y, Chen J, Chanock SJ, Zheng SL, Xu J, Thun MJ, Calle EE, Rodriguez C: Polymorphisms in angiogenesis-related genes and prostate cancer. Cancer Epidemiol Biomarkers Prev 2008, 17(4):972-977.

61. Chau CH, Permenter MG, Steinberg SM, Retter AS, Dahut WL, Price DK, Figg WD: Polymorphism in the hypoxia-inducible factor 1alpha gene may confer susceptibility to androgen-independent prostate cancer. Cancer Biol Ther 2005, 4(11):1222-1225.

62. Ollerenshaw M, Page T, Hammonds J, Demaine A: Polymorphisms in the hypoxia inducible factor-1alpha gene (HIF1A) are associated with the renal cell carcinoma phenotype. Cancer Genet Cytogenet 2004, 153(2):122-126.

63. Li H, Bubley GJ, Balk SP, Gaziano JM, Pollak M, Stampfer MJ, Ma J: Hypoxia-inducible factor-1alpha (HIF-1alpha) gene polymorphisms, circulating insulin-like growth factor binding protein (IGFBP)-3 levels and prostate cancer. Prostate 2007, 67(12):1354-1361.

64. Zhong H, De Marzo AM, Laughner E, Lim M, Hilton DA, Zagzag D, Buechler P, Isaacs WB, Semenza GL, Simons JW: Overexpression of hypoxia-inducible factor 1alpha in common human cancers and their metastases. Cancer Res 1999, 59(22):5830-5835

65. Bardos J, Ashcroft M: Hypoxia-inducible factor-1 and oncogenic signalling. Bioessays 2004, 26(3):262-269.

66. Vaupel P: The role of hypoxia-induced factors in tumor progression. Oncologist 2004, 9(Suppl 5):10-17. 
67. Zhao T, Lv J, Zhao J, Nzekebaloudou M: Hypoxia-inducible factor-1alpha gene polymorphisms and cancer risk: a meta-analysis. J Exp Clin Cancer Res 2009, 28:159.

68. Li D, Liu J, Zhang W, Ren J, Yan L, Liu H, Xu Z: Association between HIF1A P582S and A588T polymorphisms and the risk of urinary cancers: a meta-analysis. PLoS One 2013, 8(5):e63445.

doi:10.1186/1471-2407-14-950

Cite this article as: Yan et al: Association between HIF-1a C1772T/

G1790A polymorphisms and cancer susceptibility: an updated

systematic review and meta-analysis based on 40 case-control studies.

BMC Cancer 2014 14:950.

\section{Submit your next manuscript to BioMed Central and take full advantage of:}

- Convenient online submission

- Thorough peer review

- No space constraints or color figure charges

- Immediate publication on acceptance

- Inclusion in PubMed, CAS, Scopus and Google Scholar

- Research which is freely available for redistribution 